\title{
L'arc et le sang des chiens
}

The bow and dog blood

Philippe Jespers

\section{(2) OpenEdition}

Journals

Édition électronique

URL : http://journals.openedition.org/span/533

DOI : 10.4000/span.533

ISSN : 2268-1558

\section{Éditeur}

École pratique des hautes études. Sciences humaines

\section{Édition imprimée}

Date de publication : 1 avril 1984

Pagination : 65-102

ISSN : 0294-7080

\section{Référence électronique}

Philippe Jespers, "L'arc et le sang des chiens », Systèmes de pensée en Afrique noire [En ligne], 6 | 1984, mis en ligne le 05 juin 2013, consulté le 19 avril 2019. URL : http://journals.openedition.org/span/533 DOI : 10.4000/span.533 
$L^{\prime A R C}$ E T LE SANG DES C H I EN S

par

Philippe Jespers

"Que kle te donne une bonne assise au village de nos vieux morts". C'est en ces termes que s'exprime un fils aîné minyanka quand il est amené à sacrifier un chien pour les funérailles de son père. Ce sacrifice est connu dans toute la région de Koutiala sous le nom de sunbwègè yiighe ("faire sortir la mauvaise médecine de l'arc"). C'est ce rite que nous allons décrire en nous efforçant de répondre à une double question :

-- quel rapport y a-t-il entre la mise à mort sacrificielle d'un chien et une assise territoriale à trouver, pour un nouveau mort, dans la terre des ancêtres?

-- comment comprendre qu'un rite de séparation entre deux générations implique ici la mort d'un animal si proche de l'homme?

Rappelons d'abord quelle est la position du chien dans le système sacrificiel minyanka.

\section{La "valeur pituelte" du chien}

Les enquêtes ethnographiques et 1 'histoire des religions nous ont appris que la mise à mort rituelle du chien, quand elle n'était pas strictement prohibée (assimilée tantôt à un acte de sorcellerie, tantôt à un meurtre), occupait une place singulière dans le système sacrificiel. Ainsi, dans certaines sociétés voltaĩques, le chien est parfois sacrifié pendant les funérailles d'un guerrier, d'un chasseur, ou après des ruptures d'interdit. Il en va de même dans l'aire mandé 
où l'on observe des sacrifices de chien notamment dans les sociétés de chasseurs, ainsi que, en certaines circonstances, dans les grandes sociétés d'initiation telles que le Komo et le Nama. Mais de l'examen des faits, il ressort avec une netteté particulière que ces sacrifices sont presque toujours appréhendés comme étant hors du commun, liés à la dimension du meurtre ou de 1 'interdit.

Les Minyanka, en revanche, loin d'en limiter l'usage à certains moments exceptionnels, pratiquent courament cette forme de sacrifice. Elle peut être effectuée dans les circonstances les plus diverses (naissance, mariage, initiation, funérailles...) et pour des destinataires de nature variée (les entités interpellées ou propitiées sont tantôt des ancêtres de lignage, tantôt des puissances de brousse). Cela vaut aux Minyanka d'être traités avec un certain mépris par leurs voisins.

La fréquence des sacrifices de chien est à mettre en relation avec le culte du Nya. D'origine mandé, ce culte a connu en pays minyanka une diffusion telle qu'il est devenu inséparable de l'identité de ce peuple. Il existe au moins une société d'initiation du Nya par village, parfois par quartier, de sorte que tous les Minyanka peuvent être considérés comme "des enfants du Nya" ou, comme nous le suggérait récemment Y. Cissé ${ }^{1}$, "les derniers enfants du Nya". Or, c'est précisément 1e Nya qui réclame parfois de véritables hécatombes de chiens.

Un mythe que nous avons commenté dans un article précédent ${ }^{2}$ éclaire la position rituelle du chien. A l'origine, le Nya était détenu par des "êtres de brousse" qui en conservaient la puissance dans des sacs placentaires d'antilope. Au cours d'une partie de chasse, l'une de ces créatures porteuses de sacs placentaires fut égorgée par le chien d'un chasseur. De retour au village, le chien livra les secrets du Nya aux hommes et mourut sur le champ. Le chasseur qui fut le premier à être initié au Nya mourut à son tour quelques jours plus tard. C'est cette double mort inexpliquée, celle du chien et du chasseur, qui est symboliquement reproduite dans le culte sacrificiel. Deux fois 1 'an, les

1. Selon ce chercheur (communication orale à l'auteur), le culte était connu depuis 1 a fondation de 1 'empire du Ghana par les Kagoro.

2. Philippe Jespers, Contribution à I'étude'des autels sacrificiels du Nya, Systèmes de pensée en Afrique noire, Cahier 2, Le sacrifice I, 1976, pp. 111-139. 
sacs du Nya sont sortis de leur sanctuaire situé au coeur du village pour être transférés en brousse par des possédés en état de transe. Sources de vie, ces sacs contenant une quantité considérable d'autelsfétiches protègent le village des attaques des sorciers et des ennemis en même temps qu'ils favorisent la fécondité de la terre.

Ainsi, mythiquement, le chien est à l'origine de la fondation du Nya, la plus importante des sociétés d'initiation minyanka. Mais il est aussi le fondateur mythique des premiers villages. En effet, d'autres mythes, qui retracent les aventures en brousse des premiers ancêtres, évoquent un chien qui d'un flair infaillible chemine vers les lieux propices à la fondation des villages. Une de ses devises, clamée par des "bouffons sacrés" lors de la sortie annuelle du Nya, le définit comme le kuzoyèrènge, "toi qui arrêtes (fondes) le village". L'analyse des rites effectués avant l'établissement d'un village, sur un site non encore défriché, nous montre en effet que la mort sacrificielle du chien y joue un rôle aussi prépondérant que lors de 1'implantation d'un nouveau Nya. Avant de délimiter les lieux de fondation d'un village, un chasseur doit s'acquitter d'une dette sacrificielle envers les génies propriétaires de la terre. Il leur offre un chien à l'endroit qu'il a jugé propice. Les os de la victime, mélangés à un peu de terre prélevée sur le lieu où le chasseur coucha la première nuit, sont enfermés dans un pot que l'on enterre, puis que l'on recouvre d'un bloc de pisé. Ce monticule prendra le nom de kashyo, "jarre du village". Situé dans un "extérieur proche" du village, entouré d'un îlot de brousse originelle, inviolable, le kashyo apparaît comme une sorte de premier ancrage du village dans l'espace de brousse. Un culte annuel y sera rendu sous la responsabilité du "chef de terre" qui n'est autre que l'aîné des descendants vivants de l'ancêtre fondateur (presque toujours un chasseur qui a scellé une alliance avec les génies du lieu).

D'autres morts sacrificielles de chiens sont requises en différentes circonstances de la vie du village, notamment lors du procès rituel qui est suivi au moment de la fondation de la "première cuisine" et du "premier vestibule" de la première maison du village.

L'examen des thèmes mythiques relatifs au détournement de la puissance du Nya de la brousse vers le village, ainsi que l'étude approfondie des rites liés à la fondation de la société initiatique du Nya et 
à celle du village permettent de mieux comprendre la position centrale du chien dans le système sacrificiel : c'est grâce à sa mort que les hommes ont pu s'assurer ici et là des "ancrages territoriaux".

Mais cette référence au mythe ne suffit pas à expliquer complètement 1a "valeur rituelle" du chien. A bien des égards il est 1 'animal le plus proche de l'homme : c'est le "gardien de la maison", le "compagnon du.chasseur", le "compagnon du guerrier". tet il n'est pas rare qu'un homme affligé par sa mort organise à son intention un rite funéraire. Il sera alors l'objet d'un deuil et enterré avec les honneurs dus à un homme ${ }^{3}$.

Mais un fait majeur doit être pris en considération : les Minyanka ont tendance à exclure du champ sacrificiel les chiens appartenant à ce que $\mathrm{E}$. Leach appelle "la catégorie du familier" ${ }^{4}$, à savoir les gardiens de maison et les compagnons de chasse, nés le plus souvent dans la maison et acquis par voie d'héritage. La ponction sacrificielle opère essentiellement sur les animaux "non familiers". Rien n'en témoigne mieux, nous semble-t-il, que ces achats massifs de chiens auxquels on assiste un peu avant la grande fẻte annuelle du Nya. En cette période extrêmement chaude de 1'année (avri1-mai), on voit des commerçants dioula et marka pousser de véritables cohortes de chiens sur les routes qui conduisent aux marchés minyanka où ils se vendent fort cher (en 1982 : 5000 à 7000 frs maliens). C'est à cette époque que les chef́s de famille reconstituent leur stock de chiens auprès des étrangers spécialisés dans ce type de commerce afin d'éviter d'être un jour acculés à sacrifier un "familier". Il existe aussi d'autres moyens d'acquérir des chiens. Ceux-ci circulent de main en main, entre maisons, entre parents et alliés, on se les donne, on se les vend au gré des circonstances. On peut aussi s'en procurer en participant aux réseaux de coopération fonctionnant à $l^{\prime}$ intérieur des sociétés d'entraide (ton) et des sociétés d'initiation (dyo). Les membres d'une classe d'initiés du Nya

3. Bien que nous n'ayons pas poussé l'enquête assez loin pour le démontrer, $i 1$ semble que les noms donnés aux chiens par les Minyanka marquent cette proximité par rapport à 'l'humain que nous essayons ici de mettre en évidence. Il existe, en outre, un principe d'appelation qui permet à un homme de se décrire lui-même à travers le nom qu'il donne à son chien : na thoeri ("moi serré"), sigi timokoso( "moi assis tout seu1")...

4. E. Leach, L'unité de Z'homme et autres essais, Paris, Gallimard, 1980 (Bibliothèque des sciences de 1 'homme). 
peuvent se rendre en équipe de dix ou quinze sur un champ de famille ou sur un champ de village en échange d'un chien équivalent à une journée de travail.

Etant donné que les sacrifices les plus fréquents portent sur des chiens anonymes achetés à des commerçants venus de loin, on peut se demander si les Minyanka ne cherchent pas, en agissant ainsi, à atténuer le malaise qu'ils éprouveraient à tuer et à consommer un animal aussi proche de 1 'homme.

Néanmoins, il nous faut insister sur le fait que, malgré ces restrictions, le sacrifice du chien ne connâ̂t pas de limite. Quels que soient leur provenance, leur mode d'acquisition, toutes les sortes de chiens peuvent un jour être amenés sur une aire sacrificielle (dans les rites funéraires comme dans le cadre des sociétés d'initiation telles que le Nya, le Komo, le Nankon, etc.). Rien n'interdit en soi le sacrifice d'un familier. Au stade actuel de notre enquête, il ne nous est pas possible de cerner les circonstances exceptionnelles qui poussent un homme à commetre un tel acte. Cependant, ce propos d'un de nos informateurs nous semble exemplaire: "Tous les ans, j'offre au Nya de mes ancêtres un chien acheté sur un marché dioula, mais si un jour le Nya me le réclamait, je lui offrirais mon meilleur compagnon". Nous ne savons pas encore par quelles voies le Nya peut exiger une telle offrande fpar la voie de son médium possédé, porteur des sacs du Nya, ou par celle des rêves..). La mort d'un chien familier constituerait en quelque sorte un lieu limite du champ sacrificiel.

Il reste à considérer un fait essentiel : le chien apparất sur la scène sacrificielle comme un être doué d'une capacité vengeresse exceptionnelle. Il est dangereux, surchargé de puissance, contrairement aux autres quadrupèdes (ovins, bovins, caprins) qui en sont dépourvus. Les sacrificateurs l'expriment clairement : "Il est devant la mort comme un homme : il se défend". Du sang qui jaillit de son corps, on dit qu'il est "amer comme du sang humain" et de ce fait il véhicule une force vengeresse comparable à celle d'une victime de chasse ou de guerre. On comprendra mieux, dès lors, toutes les précautions que prend un sacrificateur avant d'abattre un chien. Car s'il se laissait mordre au moment de la mise à mort, il mourrait quelques jours plus tard, poursuivi par le fantôme vindicatif de sa victime. Aussi aucun Minyanka ne se risque-t-il seul au moment du sacrifice et veille-t-il à se faire 
assister ${ }^{5}$.

De tels faits tendent à rapprocher 1a "valeur rituelle" du chien, animal domestique, de celle de certains grand animaux de brousse tués à 1 a chasse. En effet, dans plusieurs sociétés voltaĩques ${ }^{6}$, le chasseur de granł gibier doit se soumettre, après l'abattage de l'animal, à des rites de purification qui ne sont pas sans rappeler ceux que subit un meurtrier pour échapper à la vengeance de sa victime. En revanche, les animaux domestiques sacrifiés ne déploient aucun pouvoir vengeur et certains traits du rite sacrificiel marquent la distance qui sépare 1 'acte sacrificateur de 1 'acte meurtrier.

Qu'en est-il donc d'un sacrifice dont la victime représente pour le sacrificateur un risque comparable à celui auquel s'expose un meurtrier ? Pour répondre à cette question, il nous faut revenir au mythe exposé plus haut. Le chien meurt au village pour avoir livré les secrets du Nya aux hommes et sa mort est suivie immédiatement de celle du chasseur. Mais l'acte de violence meurtrière dont fait état le mythe doit lui-même être mis en relation avec le danger mortel encouru par tous ceux qui, depuis la fondation du Nya jusqu'à nos jours, ont voulu s'approprier cette puissance de brousse à titre de nyatigi ("mâttre du Nya"). En effet, lorsqu'un candidat veut implanter un nouveau Nya, il se rend auprès du maître du Nya d'un autre village qui le prévient qu'un acte de cette nature entraînera à plus ou moins brève échéance sa propre disparition (à sa mort, on intégrera quelques fragments de son propre coprs aux sacs). Chaque nouvel acquéreur du Nya est comme ce chasseur qui périt pour avoir arraché les sacs à leurs véritables propriétaires de brousse (les descendants d'une première humanité déchue).

Nous sommes donc amené à nous interroger sur une série de morts dont les victimes représentent le couple chien-chasseur du mythe. Cette double mort doit être comme répétée, à chacune des reconstitutions des sacs du Nya, à travers la mort virtuelle de ceux qui prétendent s'arroger le Nya à titre de propriétaire (nyatigi) d'une part,

5. Il n'est pas rare, en outre, qu'on administre à la victime une médecine avant de 1 'abattre.

6. Cf. M. Cartry, Compte-rendu des conférences de L'année 1980-1981 à l'EPHE, Annuaire, Tome LXXXIX, EPHE (Ve section). 
le sacrifice du chien ${ }^{7}$, d'autre part.

La confrontation des rites sacrificiels avec le mythe nous permet de faire l'hypothèse qu'avec la mort du chien les Minyanka se rapprochent de cette limite que franchit le meurtrier. Nous allions maintenant essayer de comprendre ce qui est en jeu dans le sacrifice effectué lors des rites funéraires.

\section{LE RITE DU SUNBWEGE YIIGHE}

Un homme vient de mourir au village. Quelques jours après son inhumation dans la terre des "vieux morts", toute sa parenté masculine se réunit pour organiser à son intention le rite du sunbwègè yiigh he. Ce terme se décompose ainsi :

-- sun, qui est une contraction de sunta, désigne I'arc ;

-- bwègè désigne, dans le contexte de la guerre traditionnelle, une "mauvaise médecine" destinée à protéger un guerrier contre le pouvoir vengeur de sa victime;

-- yiighe est un verbe signifiant "faire sortir".

On peut donc traduire sunbwègè yiighe par "faire sortir la mauvaise médecine de I'arc". Interrogés sur la portée de ce rite qui a tous les caractères d'un rite de levée de deuil, les vieux Minyanka nous ont fait un récit que nous résumons comme suit. Un jour, quelque temps après la mort de son père, un fils aîné décida d'organiser un sunbwègè yiighe à la porte de la "première cuisine" de la maison paternelle. Son père était connu dans tout le pays pour être un grand guerrier. En faisant. "sortir la mauvaise médecine de I'arc" ayant appartenu à son père, le fils aîné répétait les gestes que ce dernier avait lui-mêne effectués à chaque fois qu'il revenait de guerre. Le sunbwègè était alors destiné à mettre le guerrier à l'abri du nyama, de la force vengeresse qu'il avait déclenchée en tuant sa victime sur un champ de bataille. Pour rappeler que son père avait

7. Les Minyanka disent qu'avant la colonisation, ils sacrifiaient également un homme fou au Nya. On peut se demander à ce propos si le fou, par ses pérégrinations agitées, n'entretient pas le même type de rapport à l'espace de brousse que le chien, ce qui fait de lui un médiateur supplémentaire entre les hommes et "la brousse du Nya". Les Minyanka le disent explicitement : le chien comme victime sacrificielle est le "second du fou". 
brisé la tête de beaucoup d'ennemis, le fils sacrifiait un chien en l'abattant à la hache et, avec les morceaux de corps de la victime, il confectionnait des "trophées de guerre". De même, quand aujourd'hui un homme de grande renommée meurt au village, il est strictement prescrit que le fils â̂né accomplisse un sunbwègè yizighe, quand bien même son père n'aurait été ni guerrier, ni chasseur, ni meurtrier.

Ce récit met d'emblée en jeu l'idée qu'un père défunt doit être traité par son fils aîné, quelques jours après son décès, comme s'il avait tué des ennemis à la guerre.

Le sunbwègè pose un problème d'ethnologie comparative. En effet, nous retrouvons dans les séquences de ce rite de deuil des opérations semblables à celles qui ont été observées ailleurs à propos des rites de purification liés à la chasse et à la guerre. On pourrait citer, dans l'aire mandé comme dans l'aire voltaïque, de nombreux cas où un tueur, mis en danger par son acte (qu'il ait tué à la guerre ou dans une autre circonstance), est traité rituellement et où intervient la mise à mort d'un chien associée à la manipulation de son arc et de son carquois. Au temps où les Minyanka pratiquaient la guerre traditionnelle, le sunwbègè était $1^{\prime}$ un de ces rites de purification. Nous reviendrons en détail sur ce point, mais nous pouvons d'ores et déjà indiquer qu'il s'effectuait obligatoirement à la porte de la "première cuisine" de la maison du tueur dès le retour triomphal de ce dernier. Comme son nom 1'indique, le sunbwègè yiighe consistait pour le tueur à "faire sortir" la redoutable médecine qu'il avait préalablement constituée à partir d'éléments du corps de la victime d'une part, de l'instrument de la mise à mort (arc, carquois, flèches) d'autre part. Cette médecine était ensuite triomphalement menée hors du village vers le "champ des ordures" ò̀, fixée sur une perche, elle était destinée à mettre la communauté à 1 'abri des forces mystiques que le guerrier avait déclenchées en fléchant sa victime sur le champ de bataille.

Or ce sont précisément ces gestes que le fils aîné est encore aujourd'hui appelé à reproduire quelques jours après le décès de son père. Les "affaires d'homme" (arc, carquois, flèches...) de ce dernier sont au centre du sunbwègè yiighe è il est assimilé de ce fait à un guerrier revenu du champ de bataille.

Comment comprendre qu'un rite de deuil emprunte sa trame à un rite de purification lié à la guerre? Ce n'est qu'au terme de cet 
article qu'une réponse à cette question pourra être esquissée. Il nous faut entrer maintenant dans le détail du rituel en commençant par une rapide présentation des acteurs et des lieux.

\subsection{L'officiant principal : le fils ainé}

Pour les Minyanka, le sunbwègè est une prescription rituelle obligatoire à laquelle nul ne saurait se dérober sans provoquer des événements qui bouleverseraient l'odre social. Si quelques semaines après la mort de son père le fils aîné ne lui a pas "rendu" le sunbwègè, le défunt est censé redescendre vers le village et y faire peser une menace de mort sur son fils d'abord (on ne conçoit pas que ce dernier puisse survivre sans avoir accompli ce devoir rituel), sur les autres parents, ensuite.

Chez les Minyanka, le fils aîné porte le nom de shyè, "premier". Malgré -- ou en raison -- des nombreux tabous qui régissent sa relation avec son père, il apparâ̂t, déjà du vivant de ce dernier, dans une position d'"héritier spirituel"8. C'est à lui que le père confie les connaissances secrètes qu'il détient (quête des racines en brousse, incantations, etc.).

Seul un fils aîné circoncis (en âge de procréer) peut remplir la fonction d'officiant du sunbwègè. Si le père meurt avant la circoncision de son enfant, c'est un neveau utérin (nalo) du défunt qui, à la demande du maître de maison, se chargera du rite. En aucun cas, la fonction d'officiant ne pourra être assurée par un membre de la génération du défunt ${ }^{9}$.

C'est donc bien la position du fils aîné comme "premier" -- il faudrait dire le premier qui ait transformé son père en géniteur --

8. Nous ne pouvons pas traiter dans le cadre de cet article du problème complexe de la transmission des biens et des charges juridiques et rituelles.

9. Notons que la règle selon laquelle le.sunbwègè ne peut être effectué que pour un homme ayant engendré au moins un fils subit de notables inflexions lors du décès d'un homme de grand renom ("maître du Nya", "mâ̂tre du Komo", "mâ̂tre de la terre"...) qui ne remplit pas cette condition minimale. De leur vivant, ces hommes ont été considérés comme des "pères" par les initiés des grandes confréries socioreligieuses dont ils ont la responsabilité. La charge du sunbwègè échoit alors à un fils aîné classificatoire qui jouera le rôle habituellement dévolu au fils réel. 
qui est en jeu dans le sunbwègè où il doit être, disent les Minyanka avec insistance, "1'égal de son père". C'est assurément l'une des questions-clefs pour l'interprétation de ce rituel.

\subsection{La "première cuisine"}

Le sunbwègè débute nécessairement au seuil du tyèrè gbo, la "première cuisine", généralement située au centre de l'espace d'habitation du gbun ${ }^{10}$, la grande maison collective. A se promener dans le dédale d'une maison minyanka, on constate qu'il existe en son centre au moins deux cuisines. Bien que I'une et 1 'autre soient construites sur le. même modèle (de plan rectangulaire) et comportent les mêmes éléments (double foyer, jarres, poteries encastrées en terre), la première, le tyèrè gbo, est réservée à l'usage des ancêtres, et la deuxième, le tyèrè, à l'usage des vivants. D'autre part, en dégageant les moments importants de la construction d'une nouvelle maison, on constate que c'est le tyèrè gbo qui est édifié en premier lieu par le fondateur (souvent un chasseur) ; c'est une sorte d'espace matriciel autour duquel prendront place les autres éléments. C'est au gbunfQlq, "maître de maison" (1e membre le plus âgé de la plus ancienne génération du segment de lignage), que revient la charge de la "première cuisine". Les cérémonies qu'il y préside sont destinées à assurer la prospérité de l'ensemble de la maison, notamment dans le cadre des cultes rendus aux jumeaux et aux ancêtres. Avant la colonisation française, le tyèrè gbo servait aussi à divers "rites de guerre" : rites de préparation des médecines et des poisons avant une expédition contre un ennemi extérieur ; rites de purification (comportant la constitution de médecines et de trophées de guerre destinés à protéger l'intégrité de la maison) au retour.

Avant même le début du sunbwègè, dès les premiers moments du cycle funéraire, le fils â̂né du défunt investit l'espace de la "première cuisine".

Les rites funéraires débutent dans 1a "première douchière". Le défunt, soutenu par des deuilleurs de sa génération et par des parents à plaisanterie (senanku), est assis nu sur un mortier renversé. Après la

10. Le terme gbun désigne à la fois : -- un groupe résidentiel formé d'un segment de patrilignage et des épouses des agnats ; -- 1'espace habité par ce groupe. 
toilette. Le "maître de maison" verse un peu d'eau sur les extrémités du corps du défunt dans 1'ordre suivant : - sur la paume de la main gauche -- sur la plante du pied droit -- sur la plante du pied gauche -- sur la paume de la main droite. Ce geste, qui dessine un signe en " $X "$ (signe de vie et de fécondité) sur le corps, marque la fin de la toilette rituelle destinée à le purifier de la souillure de la mort. L'eau qui a servi à cette opération et à laquelle il est rigoureusement interdit de toucher sera évacuée par un trou pratiqué dans le sol de $1 \mathrm{a}$ douchière. Le fils aîné entre alors en scène. I1 s'accroupit devant le corps de son père et, tenant un peu d'eau dans le creux de ses mains jointes, il saisit le sexe du défunt et lui fait subir une légère pression tout en esquissant un mouvement d'avant en arrière comme s'il voulait en extraire quelque chose. Il répète ce geste trois fois de suite en veillant à conserver l'eau dans ses mains jointes. Alors, il se lève brusquement et fuyant droit devant lui (avec l'interdiction de se retourner), il emporte cette eau jusqu'aux abords du tyèrè gbo. Après s'être déchaussé, il entre dans l'enceinte en ruine de cette "première cuisine" et verse 1 'eau dans une des poteries encastrées dans la terre. Il retourne ensuite dans la première douchière où 1 'attendent d'autres tâches.

Qu'a fait au juste le fils aîné ? D'après nos informateurs, l'eau pure qu'il porte dans la "première cuisine" est une dernière "eau de sexe" du père défunt. Contrairement aux opérations précédentes, effectuées par les autres deuilleurs, il ne s'agit donc pas là seulement d'un rite de purification. Au lieu d'être versée dans la "première douchière", lieu destiné à recueillir la souillure de la mort, l'eau est en effet transférée jusqu'à la "première cuisine" qui est au contraire un lieu très pur puisqu'un feu y est entretenu pour les ancêtres de 1 a maison ${ }^{11}$.

Seul le fils aîné a le pouvoir d'extraire du corps de son père, lavé de l'impureté liée à la mort, une part de la puissance fécondante

11. Une étude plus complète des rites funéraires, que nous ne pouvons pas entreprendre dans le cadre de cet article, dégagerait les rapports de cette séquence rituelle avec d'autres séquences du cycle funéraire, notamment celle de l'enterrement (effectué par le fils aîné dans la "première douchière") du cache-sexe du père défunt, et de l'octroi à ce dernier d'un nouveau cache-sexe nécessairement taillé dans un vêtement de couleur rouille ayant appartenu à un chasseur ou à un guerrier. 
qui sous la forme d'une trace d'eau laissée dans une poterie ancestrale ne saurait disparaître des murs du gbun.

\subsection{Le déroulement du mite}

Le sumbwègè a lieu quelques jours après les rites d'enterrement. La date est fixée par le "maître de maison". Le jour qui convient le mieux est le sèfèlè tshanga, jour réservé aux ancêtres de la maison marquant la fin de la semaine (semaine traditionnelle de six jours). Le jour interdit est le tyanbusaro, "quand le jour est amer", qui est précisément celui du marché marquant le début d'une nouvelle semaine.

Il est midi. Toute la parenté masculine est réunie au seuil de 1a "première cuisine". La participation de tous les hommes est prescrite; celle des femmes et des enfants est strictement interdite. Dès que les anciens de la maison engagent les préparatifs habituels du sunbwègè, l'espace du gbun se métamorphose, les abords de la "première cuisine" se vident de la présence des fenmes et des enfants. L'effervescence habituelle que connaît le centre du gbun, les allées et venues, les salutations, les jeux, les braillements des enfants..., tout s'estompe. Pendant toute la durée du sunbwègè, les femmes resteront cachées dans leurs cases ou dans les cours attenantes à celles-ci, sans prêter la moindre attention aux activités des hommes qui ont maintenant investi la "première cuisine" de la maison. Aucun enfant, sous peine des pires réprimandes, ne viendrait jeter un coup d'oeil de ce côté. Tout se passe comme s'il s'agissait pour les hommes d'organiser au centre de l'espace d'habitation du gbun un rite d'initiation masculine. Un ancien s'accroupit et commence par ranger tout le matériel qui servira de support aux opérations sacrificielles du sunbwègè. Le rangement répond à un schéma préétab1i. : 1'homme dispose à terre un carquois, un arc, dix flèches, trois souches de mil (arrachées précédemment dans un champ de petit mil) et une écuelle en bois, selon un axe rectiligne, orienté nord-sud, et dans le prolongement de 1 a porte de 1 a "première cuisine" (voir photo $n^{0} 1$ ). Nos informateurs sont unanimes sur la réalité symbolisée par cette série d'objets : ce sont les nami yèrè yô, les "affaires d'homme" du défunt, autrement dit les attributs de sa masculinité. nari yèrè yôx est une expression qui reviendra comme un leitmotiv dans les chants de louanges des griots adressés au mort pendant et après les opérations 
sacrificielles. Or c'est précisément l'ensemble de ces objets liés à 1a puissance du défunt qui se trouvera au coeur du rituel de la "sortie de la mauvaise médecine de 1'arc". Certains d'entre eux (1'arc, le carquois, les flèches) connotent l'activité guerrière, les autres (les souches de mil, l'écuelle en bois) l'activité agricole. Ces objets n'ont pas le même rapport au temps dans 1'espace de 1a maison : les uns sont d'un usage permanent, les autres d'un usage éphémère. Ainsi, le carquois et les flèches sont conservés d'une manière continue, généralement accrochés aux murs du "premier grenier" ou du "premier vestibule" de la maison, ce sont les éléments non périssables du sunbuègè. En revanche, l'arc, remplacé à chaque rituel, en est l'élément mobile et périssable. Cette différence de traitement entre 1'arc d'une part, le carquois et les flèches d'autre part, a des implications beaucoup plus vastes qu'on ne pourrait le supposer à première vue. Elle détermine, nous le verrons, le sens ultime des opérations sacrificielles du sunbwègè.

Après le rangement des nari yèrè yâa, un "chien rouge" fait son apparition dans le cercle des deuilleurs. L'animal est conduit à l'aide d'une laisse rigide faite d'une corde qui enserre étroitement son corps, au niveau des reins, du dos et du cou, et d'un bâton permettant de le maîtriser à distance. Observons alors que la couleur de son pelage est sans ambiguité : le sumbwègè n'accepte que des chiens "rouges", nyèrè (dans notre système des couleurs nous dirions "roux"). Eu égard au code des couleurs minyanka, on peut considérer que le rouge connote le caractère violent des opérations qui s'annoncent ici au seuil de la "première cuisine".

\section{La prière}

Tout est prêt. Les opérations sacrificielles vont pouvoir débuter. Les deuilleurs formant cercle autour de lui, le fils ainé s'accroupit devant les nari yèrè yâa de son père défunt et se prépare à réciter la prière du sunbwègè, la face tournée vers l'est. Pourquoi vers l'est ? On aurait pu s'attendre à ce qu'il se tourne vers l'ouest dans la mesure où cette direction indique ici, comme dans bien d'autres sociétés mandé et voltaiques, le point de non retour des morts et que c'est aussi à 1 'ouest (de toute façon jamais à 1 'est) que se situe par rapport au village des vivants celui des morts. Or justement il n'en est rien. 
Pour mieux faire ressortir l'importance de cet axe ouest-est qui commande le "travail de deuil" du sunbuègè, il convient de se reporter aux grands axes de la cosmogonie. L'est se dit en langue minyanka kle nygh, ce qui peut se décomposer et se traduire par : 1) nygh, "commencement" ou "derrière" ; 2) kZe, "ciel" ou "Dieu". Le terme renvoit à un commencement absolu dans I'ordre du temps et de $1^{\prime}$ espace. Autrement dit, c'est de l'est que vient toute chose, le "destin" (numepana), 1a "chance" (karesege), la "renommée" (fama), la richesse, les enfants... c'est dans une matrice céleste originelle que kle a inscrit le destin de tout être. Dès lors, quand il s'agit dans un rite de se référer à ce lieu de la production de tout être, de tout destin, on se tourne en principe vers le kle nygh, le "commencement du ciel". Pour le fils aîné, se tourner vers 1 'est de la maison paternelle revient en quelque sorte à reprendre symboliquement les choses au commencement ${ }^{12}$.

Revenons au déroulement du rite. Le fils aîné, tenant à présent le chien rouge par la laisse, se prépare à réciter la prière du sunbwègè. S'il est encore trop jeune pour en connâ̂tre les paroles, un ancien les lui dictera. Voici ses paroles :

"Vieux père (baba) voici ton animal à pattes pour faire sortir la mauvaise médecine de ton arc

prends-1e de jour prends-le de nuit.

et fais en sorte que moi aussi

je trouve un jour quelqu'un pour faire

sortir le mien (mon animal ...)

et que je puisse le voir avant ma mort

Vieux père, donne-moi un enfant

qui puisse faire sortir mon animal

Maintenant il ne faut plus que tu viennes discuter

ici avec quiconque à propos de cette chose;

elle t'a été donnée

Que kle te donne une bonne place à côté

de nos vieux morts

sinon toute chose finira"

12. Il est probable -- mais nous n'avons pas encore assez d'information sur ce sujet -- que le commencement dont il s'agit renvoie à la conception minyanka du destin prénatal. 
La prière est ici une invocation par laquelle le fils demande à son père de ne plus revenir discuter en ces lieux. baba est un mot emprunté à la langue bambara. C'est un terme d'adresse qui connote à la fois $I^{\prime}$ affection et le respect. Indiquons que cette manière d'appeler un mort (ce qui est souvent le cas quand celui-ci n'est pas encore transformé en ancêtre) est censée le rendre présent et actuel parmi les vivants.

Suit alors la formule de $1^{\prime}$ offrande sacrificielle. Le fils aîné présente la victime en ces termes : mu sunbwègè yiighe yatogo, "voici ton animal à pattes qui va pouvoir sortir la mauvaise médecine de 1 'arc". La formule se décompose ainsi :

-- mu est la marque du possessif "ton"

-- sunbwègè est la fameuse "médecine de 1 'arc" dont nous connaissons déjà 1 'étymologie

-- yiighe est un verbe signifiant "faire sortir"

-- yat 999 , la "chose à pattes", est le terme générique de tous les animaux domestiques à quatre pattes.

En assignant ici à la victime la fonction de "faire sortir la mauvaise médecine de 1 'arc", le sacrificateur la définit comme une sorte de véhicule grâce auquel la part dangereuse de la puissance du père défunt sera évacuée hors des murs de la maison paternelle. Certes, 1a prière n'explicite pas comment ce déplacement s'opère (nous le verrons dans les opérations post-sacrificielles), mais elle en indique avec précision le but. Réécoutons le fils : "Prends-le (cet anima1)... et fais en sorte que moi aussi je trouve un jour quelqu'un (un fils) pour faire sortir le mien...". Par cette formule, le fils demande presque explicitement à son père d'intervenir auprès des "vieux morts" dont on sait par ailleurs qu'ils coopèrent à la venue des enfants, et ceci annonce déjà le statut d'ancêtre du père.

Tels sont les effets escomptés du rite : le fils â̂né doit "faire sortir" hors des murs de la maison le sunbwègè de son père pour que ce dernier puisse quitter définitivement l'espace des vivants et intégrer la terre des "vieux morts" où il est appelé par kle à devenir un ancêtre. 


\section{Abattre}

Dans le sunbwègè, I'abattage du chien suit toujours le même processus : 1a victime est d'abord renversée sur le dos par deux assistants qui essaieront ainsi de la maîtriser"13. Un vieux s'écrie : "tenezle bien! tenez-1e bien!". La tension monte, car si l'un de ces hommes se laissait mordre par le chien, il mourrait dans les jours à venir, poursuivi par le nyama de l'animal. Et il va sans dire qu'aux yeux des Minyanka, le nyama d'un chien rouge est infiniment plus virulent que celui d'un chien d'une autre couleur.

Le sacrificateur s'empare d'une lourde hache. I1 est prescrit d'utiliser celle-ci non par la lame dite "bouche de hache", mais par le manche dit "tête de hache" afin d'éviter un écoulement prématuré du sang. Faisant tournoyer la hache, le fils â̂né $1^{\prime}$ abat de toutes ses forces sur le crâne de sa victime. Un ou deux coups de cette violence et la mort survient. Le choc sourd de l'instrument sur la tête du chien arrache aux deuilleurs un cri, un hé! hé! de joie et de victoire. Deux gboyq sortent de 1 'ombre. Ce sont des joueurs de harpe-1uth, instrument à trois cordes que les Minyanka utilisaient jadis pour ponctuer la parade qui suivait le retour triomphal des guerriers. I'instrument se présente comme une calebasse de grande taille (la caisșe de résonance) prolongée d'un manche à $l$ 'extrémité duquel des plaques de sonnailles méta1liques brouillent le jeu des trois cordes. Les deux gboy appuyant la calebasse sur leurs genoux, le buste oscillant d'avant en arrière, 1ancent, véhéments, un vieux rythme guerrier. Tout se passe comme si la syntaxe rituelle attribuait au choc sacrificiel le pouvoir de déclencher une musique guerrière qui accompagnera toute la suite des opérations. Au rythme du nomampè, "I'homme laid", les gboyQ adressent les premières louanges au défunt :

"Voici ses affaires d'homme nous sommes en train d'appeler nam'pè (le "quatrième fils d'homme")

13. Dans un article précédent, nous avons vu que le chien consacré à la puissance cosmique du Nya n'était pas abattu à la hache. A l'extérieur des enclos de brousse du Nya, les sacrificateurs, ramenant les pattes de 1 'animal derrière son échine, le projettent violemment à terre, lui fracassant le crâne ou la colonne vertébrale. Cf. Ph. Jespers, Contribution à l'étude des autels sacrificiels du Nya, Systèmes de pensée en Afrique noire, Cahier 2, Le sacrifice 1, pp. 111139 . 
voici ses affaires d'homme

nous sommes en train d'appeler tempe ngolo dya ("I'enfant du troisième fils de la maison de tempe")

laissez le porc-épic sinon il va tirer sur nous avec ses flèches, poule des fossoyeurs des morts

voici ses affaires d'homme

nous sommes en train d'appeler bugu nam'pè (le "quatrième fils d'homme de la case du Nya")

le porc-épic, c'est le porc-épic que nous avons tué

tourner autour du village, on est en train de tourner autour du village".

La formule nami yèrè $y \hat{\alpha}$, "voici ses affaires d'homme", constitue le leitmotiv de ces chants de louanges. Ce que vise l'expression, ce sont, bien entendu, l'arc, le carquois et les flèches, instruments de la puissance guerrière du père. La formule sera répétée plusieurs fois en alternance avec différentes formes de louanges. Nous apprenons que le défunt était :

-- nom'pè, "quatrième fils d'homme". ; dans le système des appelations minyanka, mpè est le nom ordinal désignant le quatrième fils d'un homme.

-- tempe ngolo dya, "enfant du troisième fils d'un homme de la maison de tempe" (nom du gbun); ngozo est ici un nom ordinal désignant le troisième fils d'un homme.

-- bugu nam'pè, "quatrième fils de la case du Nya" ; c'est le nom personnel du défunt. En principe un nom personnel entier se compose de deux termes : 1) du nom dérivé de l'appelation de la puissance cosmique qui a favorisé la naissance (ici, le sanctuaire du Nya) ; 2) d'un nom ordinal exprimant 1 'ordre de naissance, mpè désignant ici le "quatrième".

La déclamation des différents noms du défunt réaffirme avec emphase la position qu'il occupait sur 1 'axe de la filiation patrilinéaire ; il était le quatrième fils de son père qui était lui-même le troisième fils d'un homme de la "maison" de tempe (nom individualisé du gbun). Elle réafirme aussi qu'il est né à la faveur de la puissance cosmique du liya.

"Laissez le porc-épic sinon il va tirer sur nous avec ses flèches, poule des fossoyeurs des morts". Par cette formule, les gbqyg mettent en garde contre les piquants du porc-épic qui, assimilées aux flèches de 
la guerre et de la chasse évoquent le puissant nyama (force vitale) qui se dégage du corps d'un défunt. Mais ils reconnaissent par ailleurs que ce danger est partiellement écarté, car les fossoyeurs ont déjà sacrifié leur poule sur la terre-tombe du mort.

"Tourner autour du village, on est en train de tourner autour du village". Les gboyg indiquent ici leur intention de faire bientôt le tour du village pour apaiser puis éloigner par leurs chants et leurs danses 1'âme du défunt qui n'a plus sa place au village.

Les deux gbqyg ne cesseront désormais de chanter les louanges du défunt au rythme du "1'homme laid". Mais pour les besoins de notre description, revenons aux opérations sacrificielles.

Perforer

Le chien gît maintenant tête contre terre à côté de $1^{\prime}$ arc et du carquois. Les deux assistants qui 1 'avaient immobilisé au moment de son abattage reviennent à côté du sacrificateur pour accomplir l'acte de perforation qui se dit en langue minyanka ma pût $s \hat{u}$, "on le troue". Le chien étant couché sur le flanc droit, I'un des assistants commence par raser un cercle de poils sur le côté gauche de la gorge puis, avec force, saisit la peau de la gorge et l'étire vers l'avant. Observons que les opérations successives s'effectueront sux la partie gauche du corps de la victime. Les deux assistants redressent l'animal et présentent la gorge rasée, comme une offrande au couteau. Le sacrificateur plonge aussitôt la lame en travers de la gorge, perçant au passage la veine jugulaire d'où jaillit un flot de sang épais que les hommes s'empressent de diriger vers les trois souches de petit mil. I1 verse ensuite une part de sang dans l'écuelle en bois. Quand I'animal est vidé de la plus grande partie de son sang, les hommes le projettent brusquement à terre à côté de l'arc et du carquois.

Nous référant à la théorie "énergétique" établie par Marcel Griaule à propos du sacrifice dogon, on pourrait dire que le sacrificateur a procédé icj. à l'une des opérations les plus violentes : arracher du corps de l'animal le nyama pour le transférer par le canal du "sang amer" vers l'un des supports du sunbwègè; les trois souches de mil symbolisant 1 'après-récolte.

Remarquons que pour arriver à ses fins, le fils sacrificateur n'a pas "égorgé" la victime comme il l'aurait fait avec d'autres 
animaux domestiques (bovins, ovins, caprins, volaille). C'est là une règle absolue : pour que la mort sacrificielle d'un chien soit efficace,

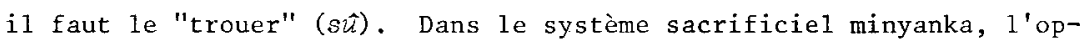
position entre deux usages du couteau est clairement établie : 1'un qui consiste à "entailler" la gorge (kor $\tilde{i})$, l'autre à la "trouer" (sî). L'examen de ce traitement différentiel du couteau donne une indication supplémentaire sur la position symbolique du chien. Le terme "percer" (sû), ainsi que les verbes formés sur ce radical, ont une portée nettement plus agressive que le terme "entailler" (kori). Le propos d'un informateur peut nous aider à mieux le comprendre : "le chien, on le perce comme par ailleurs on perce le corps d'un ennemi à la guerre ou le corps d'un animal à la chasse, avec la pointe d'une pique". Ainsi, les formes concrètes que prend la manipulation du couteau et le discours manifeste des Minyanka à ce propos font apparâttre des signifiants importants, liés à une dimension symbolique de la mise à mort ${ }^{14}$. Et tandis que le sacrificateur se prépare à d'autres opérations, les deux gboyg ( joueurs de harpe-luth) célèbrent les exploits guerriers (imaginaires) du père défunt :

"arrêtons! arrêtons (1a musique)

on dit que 1 'homme $n$ 'est plus au village quand la guerre vient le prendre s'il fait chaud, chaud et chaud (chaleur de la guerre)

le quatrième fils du troisième fils s'abrite sous le grenier vous venez de voir partir le quatrième fils d'homme quand la guerre se lève cela nous noircit le visage

..

moi je le dis, vieux fauve, vieux fauve

toi, tu as passé ta vie sans vendre quelqu'un (allusion aux captifs de guerre)

tu as passé ta vie sans démolir le village de quelqu'un tu as passé ta vie sans tuer les enfants de quelqu'un arrêtez! arrêtez! Le quatrième fils n'est plus dans le village en partant il nous a noirci le visage".

14. Que 1'acte de perforer constitue en soi un signifiant majeur du meurtre, des sacrifices de poulets effectués sur des yapèrè ("autelsfétiches") dans le cadre des pratiques de vengeance nous en apportent une confirmation indirecte. Pour donner plus de poids à son entreprise 
Comme c'est souvent le cas lors des rites funéraires, les griots usent de 1'antiphrase pour célébrer le mort. Mais les derniers vers du chant expriment sans ambiguïté ce qui vient d'être ainsi nié : les exploits guerriers du "quatrième fils". L'expression "cela nous a noirci le visage" n'est employée en effet que quand un homme, principalement un guerrier, a provoqué un important désordre au village. Ce qui a "noirci le visage". ici, c'est le départ d'un grand guerrier.

Dépecer

Une fois l'animal "troué", le sang versé sur les trois souches de mil, la dépouille reste entre les mains du fils aîné.

Tel un artisan qui va entreprendre un travail de modelage sur une matière inerte, il est maintenant penché sur le corps de la victime. Tandis que deux assistants maintiennent la tête renversée sur le sol, il reprend le couteau sacrificiel afin d'effectuer, selon un code extrêmement rigoureux, les opérations suivantes :

- $1^{\circ}$ Avec la lame bien aiguisée, il fend la commissure gauche de la bouche de la victime.

-- $2^{\circ}$ De la pointe de son couteau, il trace du haut du front au bas du museau un profond trait comme s'il voulait diviser la face du chien en deux secteurs parfaitement symétriques.

-- $3^{\circ}$ Reportant la pointe du couteau à la commissure gauche de la bouche précédemment fendue, il la fait remonter jusqu'à l'oreille gauche qu'il sectionne avec force. Puis, contournant le haut de l'oreille, il rejoint le haut du front, au point de départ de l'incision.

-- $4^{\circ}$ A $1^{\prime}$ aide du pouce, de l'index et du majeur, il saisit la peau incisée au niveau du front puis, précautionneusement, arrache une bande de peau qui laisse brutalement apparaître sur la face gauche de I'animal un derme d'une blancheur laiteuse. Que le lecteur nous excuse pour cette précision quasi-chirurgicale : si le derme est d'une telle blancheur, c'est en raison du fait que I'anima1 a été précédemment vidé de la plus grande partie de son sang.

meurtrière, le sacrificateur peut parfois "percer" la gorge du poulet comme il le ferait nécessairement avec la gorge du chien. La seule manipulation du couteau opère alors une véritable transmutation du statut de la victime : ce n'est plus un poulet mais un chien qu'il offre à $1 \mathrm{a}$ puissance neurtrière. 
Ce dépecage opère sur la scène sacrificielle le surgissement d'un être monstrueux. La face du chien se divise en deux zones verticales de couleurs opposées, le rouge et le blanc (rappelons que le chien est roux). Compte tenu du code des couleurs minyanka, on peut interpréter cette face bifide de la manière suivante : par l'une de ses moitiés, intacte et rouge, elle rappele la vie ; par l'autre, mutilée et blanche, la mort. Cette division prend une acuité encore plus grande quand on sait que, chez les Minyanka, la gauche est par excellence un symbole de la masculinité. Contrairement à ce qu'il en est chez leurs voisins (Bambara, Dogon, etc.), c'est ici la main gauche qui est la main de 1 'homme, l'oreille gauche qui est l'oreille de l'homme, le pied gauche qui est le pied de 1 'home... Ceci revient à dire que la victime sacrificielle a été chaque fois mutilée dans une partie masculine de son corps.

Interrogés sur le sens de cette triple mutilation (bouche fendue, oreille amputée, peau de la face décollée) dont la victime est successivement 1 'objet, nos informateurs nous ont apporté unanimement les précisions suivantes.

Quand un guerrier avait tué un ennemi sur un champ de bataille lointain, il ne pouvait échapper à la nécessité de fendre la bouche du cadavre et de recueillir le sang ainsi produit afin d'en crépir son fétiche de guerre portatif.

Dans un même contexte, un guerrier ne pouvait éviter de se soumettre à un autre rite de protection : il tranchait l'oreille gauche de sa victime et, fuyant droit devant lui, l'emportait jusqu'à son village. Cette oreille constituait un trophée de guerre que le vainqueur conduisait rituellement vers le "champ des ordures" de sa maison. Là, une longue perche était dressée au sommet de laquelle il fixait d'une part 1 'oreille gauche de sa victime, d'autre part son arc, son carquois et son sifflet. Cet ensemble réalisait un autel du meurtre connu sous le nom de sunbwègè, la "mauvaise médecine de l'arc", destiné à mettre toute la communauté à 1 'abri des ravages du nyama de l'ennemi.

Tel est le contexte ethnographique dans lequel se situe la trame du dépeçage de la victime. Le sacrificateur traite le chien en suivant les mêmes précautions que celles que prenait un guerrier d'antan lorsqu'il avait fléché une victime sur un champ de bataille lointain. La façon dont s'articulent les éléments de cette découpe du corps de la 
victime relève d'une sémiologie précise :

-- 1a déchirure de 1 a bouche et 1 'amputation de 1 'oreille font explicitement violence à la "parole" ; elles la coupent au sens littéral du terme en deux points de son circuit : la bouche comme lieu d'émission, l'oreille comme réceptacle de la parole. Dans la pensée minyanka, il n'y a pire violence que de fendre la bouche et/ou l'oreille de quelqu'un.

-- 1a victime a chaque fois été meurtrie dans la partie gauche de son corps. On peut penser que cette mutilation atteint de front la structure bilatérale du corps (structure par laquelle un corps humain ou animal trouve son ancrage et son équilibre vital dans 1 'espace); qu'elle frappe le côté gauche n'est pas indifférent si l'on se rappelle que c'est le côté de la masculinité. Ce qui, dans la logique de ce rite, assimile encore davantage le corps de la victime à celui d'un ennemi tué à la guerre.

Reste à dégager le symbolisme de la bande de peau décollée qui fait de la tête du chien une tête bifide. A ce stade du rite, il est encore difficile de se faire une idée de 1 'importance de cette déroupe, mais elle ne pourra que retenir davantage notre attention à mesure que nous avancerons dans la description du sunbwègè.

\section{Flécher}

Précautionneusement, le sacrificateur dépose l'oreille et la bande de peau de la victime sur deux des trois souches de petit mil qui, rappelons-le, ont recu la part la plus importante du sang sacrificiel. Ce geste réalise un étrange montage : souches de petit mil teintées de sang sur lesquelles reposent maintenant des morceaux de corps d'une victime sacrificielle appréhendée comme une victime de guerre. Une fois constitué, cet ensemble devient le noyau d'une nouvelle opération qui s'annonce comme extrêmement délicate.

Pour la première fois, le fils se tourne vers les instruments de la guerre. Du tas de flèches, il en prend deux et suivant scrupuleusement les indications d'un vieux, il les engage jumelées sur la corde de 1 'arc. Il ne s'agit pas ici de décocher les deux flèches coup sur coup comme on le ferait lors d'une partie de chasse, mais l'une et 1 'autre d'un seul coup ${ }^{15}$. Le fils â̂né dirige alors son tir vers les morceaux de corps de la victime, oreille et bande de peau, posés sur 
leurs supports respectifs de petit mil. La séquence suivante, qui culminera dans le tir des deux flèches, doit être exécutée selon un code rigoureux :

-- le tireur pointe 1'arc bandé vers l'est puis l'abaisse vers la bande peau et l'oreille.

-- le tireur pointe l'arc bandé vers 1 'ouest puis l'abaisse vers la bande de peau et l'oreille.

-- le tireur pointe 1 'arc bandé vers le sud puis l'abaisse vers la bande de peau et 1 'oreille.

-- le tireur pointe l'arc bandé vers le nord puis l'abaisse vers la bande de peau et 1 'oreille

-- le tireur pointe l'arc bandé vers le ciel puis 1'abaisse vers la bande de peau et l'oreille et cette fois, décoche les deux flèches sur la bande de peau et 1 'oreille (voir photo $\mathrm{n}^{\circ} 2$ ). A cet instant précis, un deuilleur qui s'était emparé d'une "flûte de guerre" fait retentir deux longs sifflements qui répondent ponctuellement au double fléchage de la cible.

La première opération est maintenant terminée; une seconde commence. Suivant toujours les indications des vieux - le rite prend la tournure d'une véritable initiation -, le fils aîné s'accroupit près de l'oreille et à l'aide du couteau sacrificiel la découpe selon une ligne anatomique qui sépare le "pavi1lon" (nu wèyè,"pavillon de la tête") de la "base" (nu wi, "trou de la tete"). La division de l'oreille implique alors une redistribution des morceaux de corps de la victime. Le fils aîné pose :

-- sur la première souche de mil, la bande de peau et le pavillon auriculaire;

- sur la deuxième souche de mil, celle du milieu, la base de l'oreil$1 \mathrm{e}$;

-- sur la troisième souche de mil, il ne dépose rien.

Sur la deuxième souche de petit mil devenue le support de la base de l'oreille, le fils aîné doit se livrer à une nouvelle opération. Reprenant deux autres flèches, et les jumelant, il recommence les mêmes opérations de tir, mais dirigées, cette fois, vers la base de

15. Cette manière de flécher s'articule peut-être sur certains mythes où 1 'on voit un héros chasseur s'en prendre à un animal en décochant de son arc magique des flèches jumelées. 
1'oreille, non sans avoir d'abord pointé l'arc bandé et les deux flèches jumelées vers les six points de l'espace. Retentissent alors deux longs sifflements.

L'officiant doit maintenant retirer les deux paires de flèches des morceaux de corps posés sur les deux premières souches de petit mil pour les y replanter aussitôt en les croisant en forme d'un " $X$ ". Les flèches jumelées sont ainsi transformées en "flèches-signes" (voir photo $\mathrm{n}^{\circ} 3$ ).

On obtient alors la composition suivante :

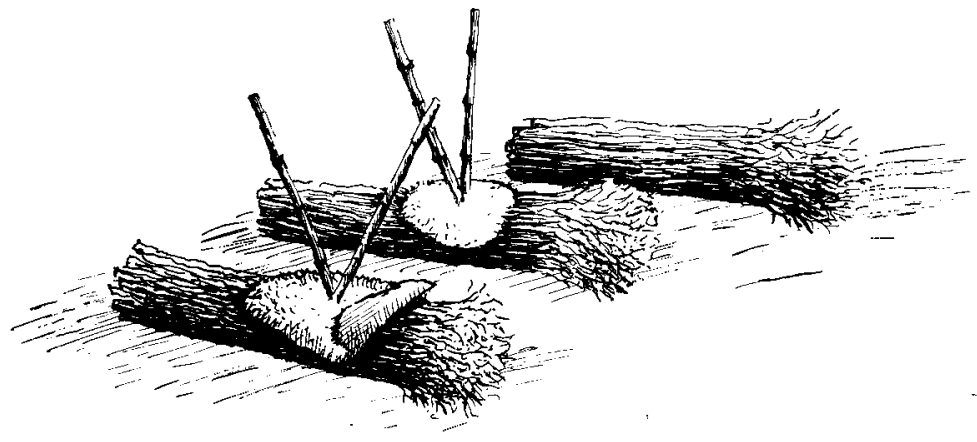

fig. 1

trophées de guerre

(région des Koromo)

Cette composition comprend dans 1 'ordre :

-- une première souche de petit mil teinté de sang et supportant une bande de peau (prélevée de la face gauche du chien) + un pavillon auriculaire (prélevé de l'oreille gauche du chien) + deux flèches disposées en " $\mathrm{X}$ "

-- une deuxième souche de petit mil teinté de sang et supportant une base d'oreille (prélevée sur 1'oreille gauche du chien) + deux flèches disposées en " $\mathrm{X}$ ".

-- une troisième souche de petit mil teinté.de sang, ne supportant rien. 
Comment interpréter cette étrange composition ? Au stade présent de notre enquête, il est encore difficile de s'en faire une idée précise. Néanmoins, la thèse fondamentale des Minyanka, inspirée des récits de guerre de leurs ancêtres, est que ce traitement des morceaux de corps de la victime renvoie à une science magique liée à la guerrẹ et à la maîtrise du territoire. Rappelons les faits. Lorsqu'un homme réintégrait son village après une bataille victorieuse, il ne pouvait échapper à la nécessité de confectionner au seuil de la "première cuisine" de sa maison une.médecine à partir d'éléments empruntés d'une part à des portions du corps de sa victime (par exemple,' l'oreille gauche ou le crâne), d'autre part à ses propres armes (son arc ou quelques flèches). La médecine ainsi constituée faisait office de trophée de guerre. Médecine ou trophée, le but était de protéger la communauté des forces vengeresses déclenchées par l'acte meurtrier. Bien que la connaissance des Minyanka dans ce domaine soit devenue lacunaire et incertaine, les vieux sont encore en mesure actuellement de déchiffrer quelques-uns des gestes et des signes de ces pratiques guerrières transférées depuis des temps immémoriaux dans les pratiques de deuil. Nous avons pu dégager les interprétations suivantes :

-- 1 'acte de présenter $l^{\prime}$ arc bandé avec les flèches jumelées aux six points de l'espace renvoie, très certainement, à une technique destinée à maintenir ou à renforcer dans son intégrité un territoire, un village, une maison. "Que la guerre nous surprenne de l'est, de l'ouest, du nord, du sud, nous défendrons notre territoire (fele)". Cette phrase énoncée lors des rites funéraires prend tout son sens quand on comprend que la cible des deux flèches jumelées du sunbwègè (les morceaux de corps de la victime) n'est autre que le substitut symbolique de morceaux de corps d'un ennemi tué à la guerre. Ce que nous montrent ces gestes de l'archer, bandant son arc aux six directions de l'espace, c'est que le meurtre implique après coup un travail rituel de réinscription du meurtrier dans l'espace de son gbun.

-- l'acte de disposer les flèches en forme de " $\mathrm{X}$ " sur les morceaux de corps de la victime aboutit à la constitution d'un signe bien connu dans toute l'aire mandé : c'est un signe de mâtrise du territoire mais aussi un signe de fécondité (ou un symbole de la création en mouvement). Ceci ne doit pas surprendre car le pouvoir de procréation est ici étroitement lié au pouvoir de la guerre et de la chasse. 
- le fait de ne rien déposer et de ne rien flécher sur la troisième souche de petit mil est encore un de ces "signes" que les Minyanka interprètent : cette troisième souche est le lakharishu, le "mil de 1'apaisement", apaisement qui suit normalement une période de trouble dont la guerre et le meurtre ont été les générateurs.

Si cette interprétation de quelques signes et gestes du sunbwègè est exacte, on peut considérer qu'elle renvoie à une problématique de la guerre et du territoire. Pour s'en persuader, il suffirait de prendre en considération quelques variantes du sunbwègè. Rappelons d'abord que le territoire minyanka était, avant la colonisation française, divisé en plusieurs confédérations villageoises au sein desquelles les villages s'unissaient en vue d'une d'une défense collective. Il va de soi qu'en ces temps-1à, c'est la violence guerrière qui permettait à chacune de ces confédérations de maintenir son indentité. Un champ d'enquête s'ouvre ici pour tenter de repérer si. les variantes du sunbwègè ne s'expliquent pas précisément en fonction de cette ancienne division territoriale.

Si d'une région à l'autre on constate que l'oreille gauche du chien est toujours amputée, d'autres parties du corps de la victime peuvent être traitées de manières différentes. Ainsi dans la région septentrionale, dans la confédération des Sanien (les "peaux rouges"), c'est la calotte crânienne qui est découpée comme une sorte de scalp ; dans les confédérations du centre, celle des Sono (les "perroquets") et celle des Koromo (les "gens du Korè"), c'est, comme nous venons de le voir, la bande de peau de la face gauche qui est décollée; dans une confédération du sud, celle des Fashien (les "gens de la prairie"), c'est un morceau de queue qui est amputé...

Mais les variantes rituelles ne portent pas seulement sur la nature des portions de corps prélevées sur la victime, elles portent aussi sur la façon dont sont assemblés les morceaux de corps et les flèches de la guerre.

Donnons-en un exemple et commentons-1e. Dans la confédération des Fashien (les "gens de la prairie"), le fils aîné, pour réaliser la "mauvaise médecine de guerre" de son père, procède à 1 'assemblage suivant : il dispose à terre trois tiges de bambou à I'extrémité desquelles il dépose transversalement trois tiges de petit mil représentant des flèches de guerre. A l'aide de fibres végétales, il ligature un groupe 
de trois tiges de mil au sommet de chaque tige de bambou en intégrant au premier ensemble l'oreille gauche du chien et au second, sa queue. La composition se présente ainsi :

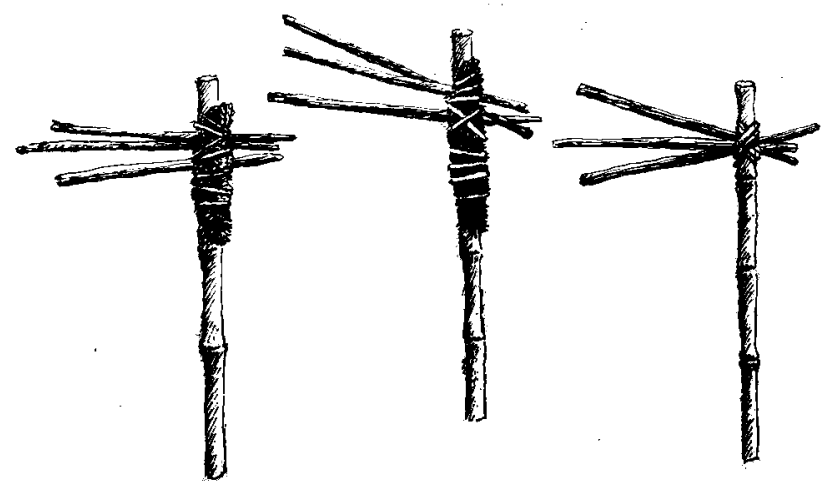

fig. 2

"trophées de guerre"

(région des Fashien)

Cette composition réalise une médecine (ou trophée) de guerre qui, si elle est fonctionnellement éruivalente à celle qui est confectionnée chez les Koromo (cf. supra fig. 1), en est morphologiquement distincte. Ceci incite à risquer $I^{\prime}$ hypothèse qu'une opposition entre deux confédérations villageoises est ici exprimée au moyen de trophées de guerre différents. Néanmoins, des règles de composition communes se dégagent aisément :

-- les trophées comportent toujours des morceaux de corps du chien symbolisant une victime de guerre;

-- les trophées vont toujours par trois (le choix d'un nombre mâle indiquant sans doute que l'impulsion meurtrière est du ressort de la masculinité) ;

-- on trouve nécessairement sur les deux premiers trophées des morceaux de corps du chien (oreille, peau de la face, calotte crânienne, queue) combinés avec des flèches de guerre;

-- le troisième trophée ne comporte jamais de morceaux de corps de la 
victime, il symbolise le temps de la paix qui suit normalement le temps de la guerre;

-- chaque trophée comporte nécessairement des éléments émpruntés au mil. Une classification des trophées de guerre par confédération villagoise est ainsi parfaitement concevable, mais elle ne nous paraît pleinement réalisable qu'à la condition de mener des enquêtes systématiques dans 1 'ensemble du territoire minyanka. Interrogés sur ces trophées de guerre, nos informateurs semblaient dire qu'ils avaient eu pour but la constitution d'une sorte d'héraldique par laquelle des groupes villageois pouvaient $j$ adis se distinguer les uns des autres sur le territoire. Il est alors remarquable de constater que cette différenciation territoriale au moyen de trophées de guerre est réactualisée dans le cadre d'un rite funéraire. Les confectionner à la porte de la "première cuisine", c'est pour les deuilleurs réaffirmer leur identité par rapport à une unité territoriale ancestrale.

\section{Sortir le sunbwègè}

Nous venons de voir comment le fils aîné a reconstitué la "médecine de guerre" de son père défunt. Il lui reste maintenant à effectuer une opération capitale, "sortir" cette redoutable médecine hors des murs de la maison.

On procède d'abord à la sortie du corps de la victime. De jeunes gaillards l'emportent en la tirant par une patte hors des murs du gbîn. Paradoxalement, la victime ne sera pas cuisinée en ce lieu dit de "première cuisine" où elle a été successivement abattue, perforée et dépe-

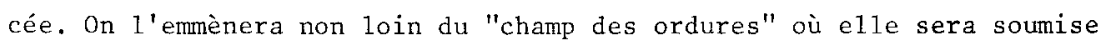
à la flame d'un premier feu qui contractera et noircira toute la surface de son corps. Et tandis qu'on éloigne la victime, les gboyq enjoignent aux deuilleurs de quitter la "première cuisine" :

"le quatrième fils d'homme nous allons 1 'accompagner au dehors pour le laisser accueillons-le au champ accueillons-le au champ".

Le fils enlève alors la corde de l'arc qu'il enroule sur le bois de l'arc, nuis abandonne l'instrument désarmé sur le sol. Il prend de 
la main gauche (la main de l'homme) le premier trophée de guerre de son père pendant qu'un deuilleur lui attache le carquois à l'épaule droite. I1 se prépare ainsi à quitter la "première cuisine".

Un cortège rigoureusement ordonné se forme derrière le fils aîné: -- un premier frère du défunt le suit les mains vides;

-- le deuxième fils suit le premier frère en tenant à la main gauche le đeuxième trophée de guerre;

-- un deuxième frère suit le deuxième fils les mains vides;

-- le troisième fils suit le deuxième frère en tenant à la main gauche le troisième trophée.

Suivent alors dans un ordre moins systématique des parents du défunt, portant qui un fusil, qui une daba (houe), qui un fagot de bois. L'ordre qui régit cette colonne de deuilleurs met en jeu une mise en alternance très stricte - comme une sorte de tissage - entre les membres masculins de deux générations distinctes. Si nous comparons la position des fils du défunt avec celle de ses frères, le contraste apparaît saisissant. Ce sont les fils qui portent les trois trophées de guerre du défunt et $c^{\prime e s t ~ u n ~ m e m b r e ~ d e ~ l a ~ g e ́ n e ́ r a t i o n ~ d e ~ c e ~ d e r n i e r ~ q u i ~}$ vient chaque fois s'intercaler entre eux, les mains vides. Dans ce cortège où se trouve privilégiée la dimension guerrière, 1'importance de la position des fils ne saurait nous échapper : ce sont eux qui héritent des insignes de la puissance guerrière de leur père ou, plus exactement, ce sont eux qui, en faisant "sortir" ces trophées de $1 a$ maison paternelle, prennent une place imaginaire dans une lignée héroīque.

La colonne des deuilleurs s'engage à présent vers l'extérieur la maison, les deux gboyg scandant le rythme de cette marche qui ressemblerait à une marche triomphale si les hommes n'étaient aussi graves et silencieux. La maison est alors désertée par les femmes et les enfants. Les gboyq chantent des couplets à la gloire du défunt :

"Homme très poilu, très poilu

l'enfant du troisième fils de la maison de tempe était très poilu.

Quand éclate la guerre nous quittons le village Cela nous noircit le visage". 
On arrive ainsi près de la sortie du gbun non loin du kunyugbo, la "grande porte" de la maison. Généralement surmonté d'un fronton majestueux, le kunyugbo est par excellence le lieu de passage de l'intérieur vers l'extérieur. Rappelons que c'est par cette porte que, quelques jours auparavant, le défunt a quitté définitivement le monde des vivants, attaché aux "trois bois", la civière des morts. Lieu charnière entre le monde des vivants et le monde des morts, le kunyugbo est aussi un lieu important du culte rendu aux ancêtres. Si l'on se réfère aux moments importants de la construction d'une nouvelle maison, on constate que cette "grande porte" n'est érigée que deux ou trois ans après la levée de deuil du fondateur et plus précisément quand celuici a acquis le statut d'ancêtre.

On ne peut alors manquer d'être frappé par le fait que les fils du défunt n'empruntent pas le kunyogbo pour "faire sortir" (yiighe) la "mauvaise médecine". (sunbwègè) du père défunt. Un homme étranger au lignage est assis à califourchon sur le haut du mur de clôture attenant au kunugbo ${ }^{16}$. Quand le fils aîné arrive à sa hauteur, il lui tend le premier trophée de guerre et le carquois. Ainsi est-ce les mains vides qu'il franchit la "grande porte" pour récupérer ensuite de l'autre côté la charge qu'il avait confiée à l'étranger (voir photo $n^{\circ} 4$ ). Le même scénario se reproduit pour les deux autres fils porteurs des deux autres trophées. Quant aux frères du morts, ils passent sans problème par la "grande porte" car ils ne portent pas les trophées de guerre attribués au défunt.

Comment comprendre que pour la "mauvaise médecine de 1'arc" du défunt il n'y ait point d'autre passage que les murs de clôture de la maison. D'où vient la nécessité de "faire sortir le sunbwègè" et précisément de cette manière-1à, aussi près de la "grande porte" ? A ce propos, les Minyanka disent ceci : "on fait sortir le sunbwègè pardelà les murs, car on sait bien qu'après son décès le défunt n'a plus besoin d'aucune porte pour revenir hanter la maison; ses âmes volètent au-dessus des murs". Ils ne dissimulent pas la crainte qu'ils éprouvent

16. Soulignons que si les murs attenants à la "grande porte" sont ébréchés, on dit quand même que les "affaires du père" passent par dessus le mur. C'est ainsi que lors d'une première enquête, j'ai pu penser un peu vite que le sunbuègè d'un défunt sortait par une brèche, la scène que j'observais se déroulant près d'une "grande porte" en ruine. 
devant le retour du double (ja) du mort, ainsi que 1'angoisse que leur inspire la toute-puissance des forces vitales (nyama), lesquelles peuvent rester comme rivées, ancrées aux personnes, aux objets, aux lieux de la vie quotidienne. Tout se passe comme si. ce spectacle d'une médecine larguée par-dessus les murs de clôture de la maison, offert à celui qui est en train de chercher sa place dans la terre des vieux morts, venait sanctionner une parole de rupture que le fils avait déjà clairement énoncée dans la prière sacrificielle : "Vieux père, il ne faut plus que tu restes ici discuter avec quelqu'un à propos de cette chose, elle t'a été donnée; que kle te donne une bonne place chez nos vieux morts sinon toute chose finira pour nous". A ce stade du rituel, le sunbwègè prend la tournure d'un véritable rite de passage. On pourrait dire : la "mauvaise médecine de l'arc" sort et avec elle la puissance guerrière du défunt qui, si elle restait "en trop" dans les murs de la maison, menacerait les fondements de celle-ci.

Parvenu ainsi hors de la maison paternelle sans avoir fait passer la "mauvaise médecine de 1'arc" par la "grand porte", le fils aîné engage maintenant la colonne des deuilleurs à faire trois fois le tour du village. La colonne accélère quelque peu le pas pendant que les gboyg ne cessent de chanter des couplets à la gloire du défunt :

\section{"Arrêtez! Arrêtez!}

le quatrième fils d'homme n'est plus dans le village

le quatrième fils d'homme est parti à la guerre

En partant il nous a noirci le visage.

Je suis allé dans la chambre à coucher du quatrième fils d'homme

la chambre à coucher m'a dit :

il était là, va demander au grand vestibule où il est

le grand vestibule m'a dit :

passe et va demander à la première cuisine où il est

moi je suis passé et je lui ai demandé

la première cuisine m'a dit :

il était là, passe et va demander à la première douchière

la première douchière m'a dit :

passe et va demander aux gens qui se trouvent sur la place

je suis allé le demander aux gens de la place

les gens de la place m'ont dit:

il était là, va demander à la grande porte où il est

j'ai été le demander à la grande porte

et la grande porte $\mathrm{m}^{\prime a}$ dit :

ici on I'a balancé trois fois, va demander au chemin du cimetière où il est 
j'y suis allé et j'ai demandé où il était

le cimetière m'a dit :

il était là, va demander à 1 ' "endroit où paît le bétail" (le cimetière des petits enfants)

1 ' "endroit où paît le bétail" m'a dit :

on l'a couché ici et on l'a salué,

passe et va demander à la tombe où il est

je suis allé le demander à la tombe

et la tombe m'a dit :

on 1 'a couché ici".

(les gboyg interrompent leur chant pour lancer un cri de victoire puis ils reprennent)

"les morts m'ont chauffé le visage

quand un homme meurt, sa tête change

Arrêtez! Arrêtez! (la musique)

Abandonnons, abandonnons nos harpes-1uths

pour aller enlever les termites

le quatrième fils d'homme,

vous aviez mis votre confiance en lui,

le village des morts 1 'a pris

cela nous a noirci le visage

beaucoup de gens ne blanchissent pas de 1 a tête

la maladie du sommeil 1 'a pris

il a refusé la mort chaude

tout homme a peur de la mort

la mort nous noircit le visage

je ne suis pas un homme, je ne suis pas un homme".

Cette longue litanie qui ponctue la marche des deuilleurs autour du village rappelle le long parcours que le corps du défunt a effectué le jour de son enterrement, de la case mortuaire jusqu'à la tombe. C'est une sorte d'anamnèse énumérant tous les lieux de la maison où $1 \mathrm{e}$ défunt s'est arrêté (première douchière, première cuisine, premier vestibule, cimetière des enfants ...). Conçue comme une conversation avec chacun de ces lieux, cette litanie entend réaffirmer que le "mort n'est plus dans le village".

L'autel du meurtre

Après avoir fait trois fois le tour du village, 1a colonne des deuilleurs s'achemine vers le kafugo, sorte de monticule qui recueille 
les ordures ménagères à quelques mètres des murs de la maison. L'importance d'une maisonnée se reconnâ̂t tout de suite à la taille de son kafugo ou champ des ordures, généralement situé dans l'axe de la "grande porte".

On contourne également trois fois le kafugo, à la suite de quoi les trois fils déposent les trophées de guerre sur le monticule. Un homme plante ensuite au milieu une longue perche tridentée en bois de néré qui va former l'axe d'un autel. Il installe en haut de la perche, comme sur une petite plate-forme surélevée, les trois trophées de guerre (souches de mil teintées de sang sur lesquelles ont été fixés avec des flèches des morceaux de corps du chien); juste en dessous, il dispose la flûte et un peu plus bas, le carquois.

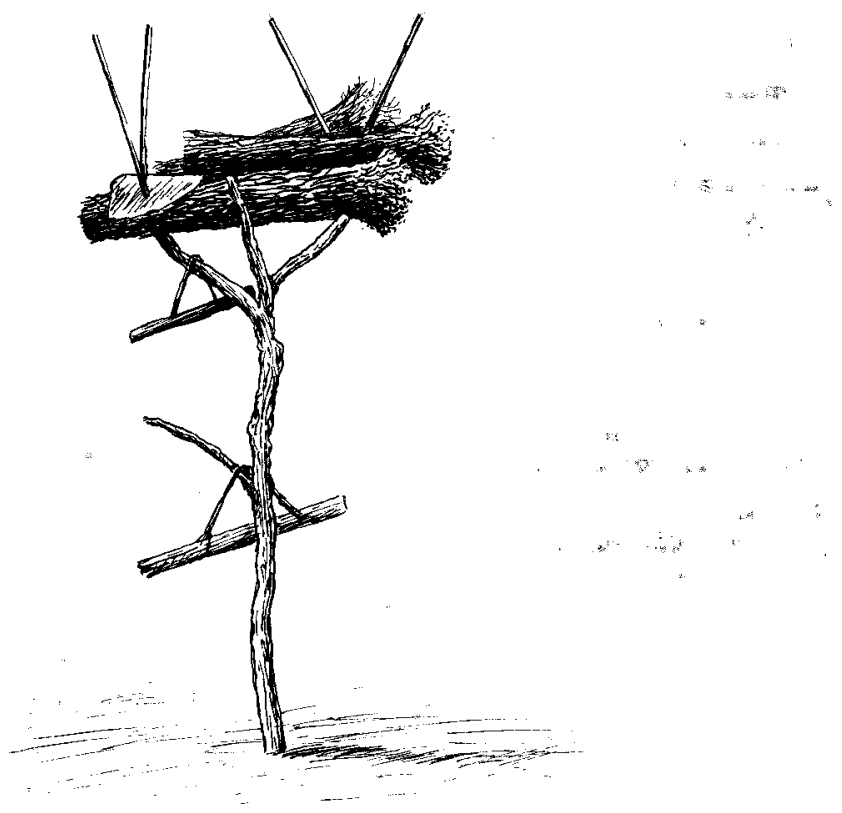

fig. 3

Autel du meurtre

Comment ne pas reconnaître dans cette composition 1'exacte réplique de ces "autels du meurtre" que les Minyanka d'antan dressaient sur le champ des ordures de leur maison après leur triomphe meurtrier. A 
la place de l'oreille gauche d'un ennemi, on trouve l'oreille gauche de la victime sacrificielle.

Regroupés devant "1'autel du meurtre", tous les hommes se recueillent pendant de longues minutes, la face tournée vers l'est. Remarquons que les deuilleurs se situent à nouveau dans I'axe ouest-est qui avait présidé aux opérations sacrificielles au seuil de la "première cuisine". Après ce temps de pause, les deuilleurs reforment une colonne et, un à un, ils vont verser une petite somme d'argent ou de cauris dans un fond de calebasse que les gboyg ont déposé préalablement au pied de l'autel. Ce geste d'offrande marque la fin des opérations sacrificielles pour les hommes.

Alors, venant du village, trois femmes gémissantes, la tête et le buste penchés en avant, s'approchent du monticule. Ce sont les deux veuves du défunt conduites par une femme, veuve du dernier défunt en date de cetce maison. La première veuve dépose à même l'entassement d'ordures 1 'arc de son mari que le fils aîné avait précédemment abandonné à la porte de la "première cuisine". Devant l'autel, se plaçant dans 1 'axe ouest-est des homes, elle chante et pleure (voir photo $n^{\circ}$ 5) :

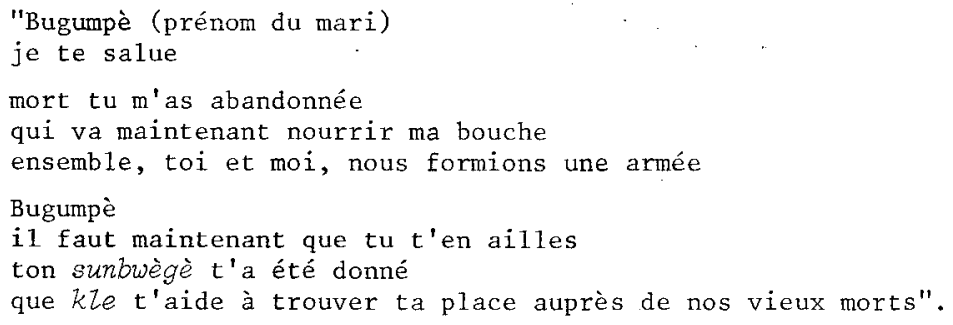

Après cet adieu, trois femmes versent également quelques pièces de monnaie et de cauris dans la calebasse des offrandes puis s'en retournent au village.

En observant $l^{\prime}$ autel du meurtre, on remarque que l'arc qui avait servi à flécher les parties du corps de la victime est maintenant désarmé et abandonné aux ordures au pied de cet autel. Que le sunbwègè yiighe marque 1 'idée d'un abandon sans retour, rien ne l'illustre mieux que le traitement de l'arc par le fils d'abord, par la veuve ensuite. Nous verrons plus loin ce qu'il adviendra de cet arc qui est ici comme 
dans bien d'autres sociétés le symbole de la puissance sexuelle et guerrière d'un homme.

Après le départ de la veuve, un grand silence envahit tout 1'espace. Les gboyg ramassent l'argent et les cauris recueillis dans la calebasse et rentrent au village. La colonne des deuilleurs, reconstituée comme au départ, quitte également les lieux. Arrivés au seuil de la "grande porte", les hommes, silencieux, s'asseyent pendant de longues minutes, I'air grave, la face tournée cette fois vers le village des morts. Par ce geste, la communauté des hommes entend signifier au défunt qu'il doit désormais se tenir à bonne distance des vivants.

La journée s'achève par la consommation de la victime. Celle-ci a d'abord été fumée dans une brousse proche du village et ensuite bouillie dans une jarre encastrée dans un angle extérieur des murs de clôture de la maison. Le corps de la victime n'a pas été dépouillée de sa peau (contrairement à ce qu'il advient aux autres animaux domestiques), mais soigneusement rasé au couteau, ce qui lui donne étrangement 1 'aspect d'un cadavre humain. Après la cuisson, on procède au repas rituel $^{17}$. La distribution des parts de l'animal ne se fait pas ici selon les mêmes règles que celles qui sont suivies lors d'autres rites sacrificiels du cycle funéraire. Pendant tout l'après-midi, dans les cours, dans les vestibules, on voit des hommes échanger et consommer des morceaux de la victime. Les os seront jetés, à l'exception des côtes que les anciens vont se réserver afin de confectionner des médecines destinées au traitement des affections séniles, en particulier le rhumatisme.

Quand la nuit tombe, la victime est entièrement consommée, 1a communauté se sent rassurée : "le mort ne reviendra pas!". Sur le champ des ordures, ses "affaires d'homme" se dressent maintenant dans la grande nuit des morts.

La rentrée du sunbwègè

Pendant six jours le sunbwègè du défunt reste suspendu à sa perche, les morceaux de corps du chien offerts aux rapaces du ciel. Le

17. Nous n'avons trouvé dans les commentaires de nos informateurs aucune indication suggérant qu'on mangerait ici symboliquement la chair du mort. Du reste, comme nous 1 'avons vu, la victime a été traitée comme le corps d'un ennemi. 
septième jour, toute la parenté masculine se retrouve au "champ des ordures" pour participer au rite du sunbwègè lènge, la "rentrée de la mauvaise médecine de 1 'arc".

Un membre de la génération du défunt commence par opérer un véritable démantèlement de 1'"autel du meurtre". Il range sur le côté le "sifflet de la guerre", le carquois et les quatre flèches nettoyées des débris de chair de la victime, et il regroupe au sommet du monticule ce qui reste de l'autel. La branche de néré qui a servi d'axe est découpée en sept morceaux qui sont déposés sur les trois souches de petit mil. Enfin l'officiant prend l'arc débandé et désarmé et le jette sur I'autel en morceaux.

Le fils aîné entre alors en scène. Tenant dans la main gauche un $\operatorname{coq}$ rouge, la face tournée vers l'est, il s'adresse à son père défunt en ces termes :

"vieux père voici ton sunbwègè lènge kolo ("coq pour la rentrée de ta mauvaise médecine de l'arc")

prends-le de jour

prends-le de nuit

et $\mathrm{fais}$ en sorte que moi aussi je puisse un jour trouver quelqu'un pour rentrer le mien

il ne faut plus maintenant que tu reviennes ici pour en faire un coq de bavardage;

il t'a été donné

que kle te donne une bonne place à côté de nos vieux morts sinon toute chose finira".

Après cette prière qui fait écho à celle qu'il avait prononcée quelques sept jours auparavant au seuil de la "première cuisine", le fils aîné égorge le coq.rouge et fait couler son sang sur les débris de 1 'autel du meurtre.

Tandis qu'il déplume la victime, un deuilleur de la génération de son père allume un feu qui fait bientôt disparâ̂tre les débris de 1 'autel du meurtre dans une épaisse fumée. Tous les deuilleurs assistent à cet autodafé auquel seuls le carquois, les flèches et la flûte de guerre échappent. Après cette destruction, un homme rassemble soigneusement les cendres et va les enfouir au somnet du monticule d'ordures. La colonne des deuilleurs se reforme et c'est au fils aîné que revient 1'obligation de transporter ce qui reste de la "mauvaise 
médecine de $I^{\prime a r c " ~ d e ~ s o n ~ p e ̀ r e . ~ T e l ~ u n ~ g u e r r i e r ~ q u i ~ r e v i e n d r a i t ~ d ' u n e ~}$ guerre sans son arc, il porte sur l'épaule droite le carquois contenant les quatre flèches et la flûte de guerre. I1 réintègre la maison paternelle sans en franchir la "première porte" et, effectuant un geste qui se perpétue identique à 1ui-même depuis des générations, i1 accroche le carquois et la flûte de guerre au mur du "premier grenier". C'est par ce geste -- et par la consommation de la victime -- que s'achève la "rentrée" du sunbwègè.

La conservation du carquois et du sifflet contraste ainsi étrangement avec la destruction de I'arc : c'est là un fait permanent de ce rite de deuil et que ne démentirait pas une analyse systématique de ses variantes ${ }^{18}$.

Le double mouvement de "sortie" et de "rentrée" de la "mauvaise médecine de l'arc" aboutit ainsi à une sorte de "tri" de ces objets que les deuilleurs nommaient si bien au début du rite les "affaires d'homme" du défunt. La souillure liée à la mort du père a été balayée à l'extérieur des murs de la maison paternelle grâce à la mort sacrificielle du chien, et c'est à la suite de ce sacrifice que le fils â̂né a pu réintroduire dans l'espace de la maison ce "quelque chose" qui semble lié intrinsèquement à la puissance guerrière de son père : 1e carquois. Cette différence de traitement devrait ainsi nous conduire à nous demander plus généralement sur quelle conception des rapports entre vivant et mort, pur et impur, mortel et immorte1... les Minyanka fondent une telle partition des "affaires d'homme" d'un défunt. Ils affirment très souvent que le carquois est cette chose propre et pure, contrairement à l'arc qui est une chose sale et impure, hors d'usage,

18. Dans la région des Fashien, le fils aîné revient du champ des ordures avec l'arc et le carquois de son père défunt. Mais pour réintégrer les murs de la maison paternelle, il ne peut échapper à la nécessité de détruire, du moins en partie, 1 'arc de son père. Utilisant les rebords supérieurs de son propre lit posé verticalement à quelque mètres de la "grande porte", il va devoir effectuer les opérations suivantes : 1) décapiter sans parole et d'un coup de couteau rapide un coq rouge, 2) jeter la victime vers la brousse, 3) dégarnir l'arc en lui arrachant la corde, 4) jeter la corde vers la brousse. Au terme de cette double destruction, il se rend au "vestibule" de la maison où, sous le regard des vieux, il ajuste une nouvelle corde à l'arc. Ensuite, arc et carquois seront accrochés au mur du "premier grenier". On ne peut pas dire que ce soit l'arc du père qui revient dans la maison puisqu'une nouvelle corde y a été ajustée par le fils aîné. 


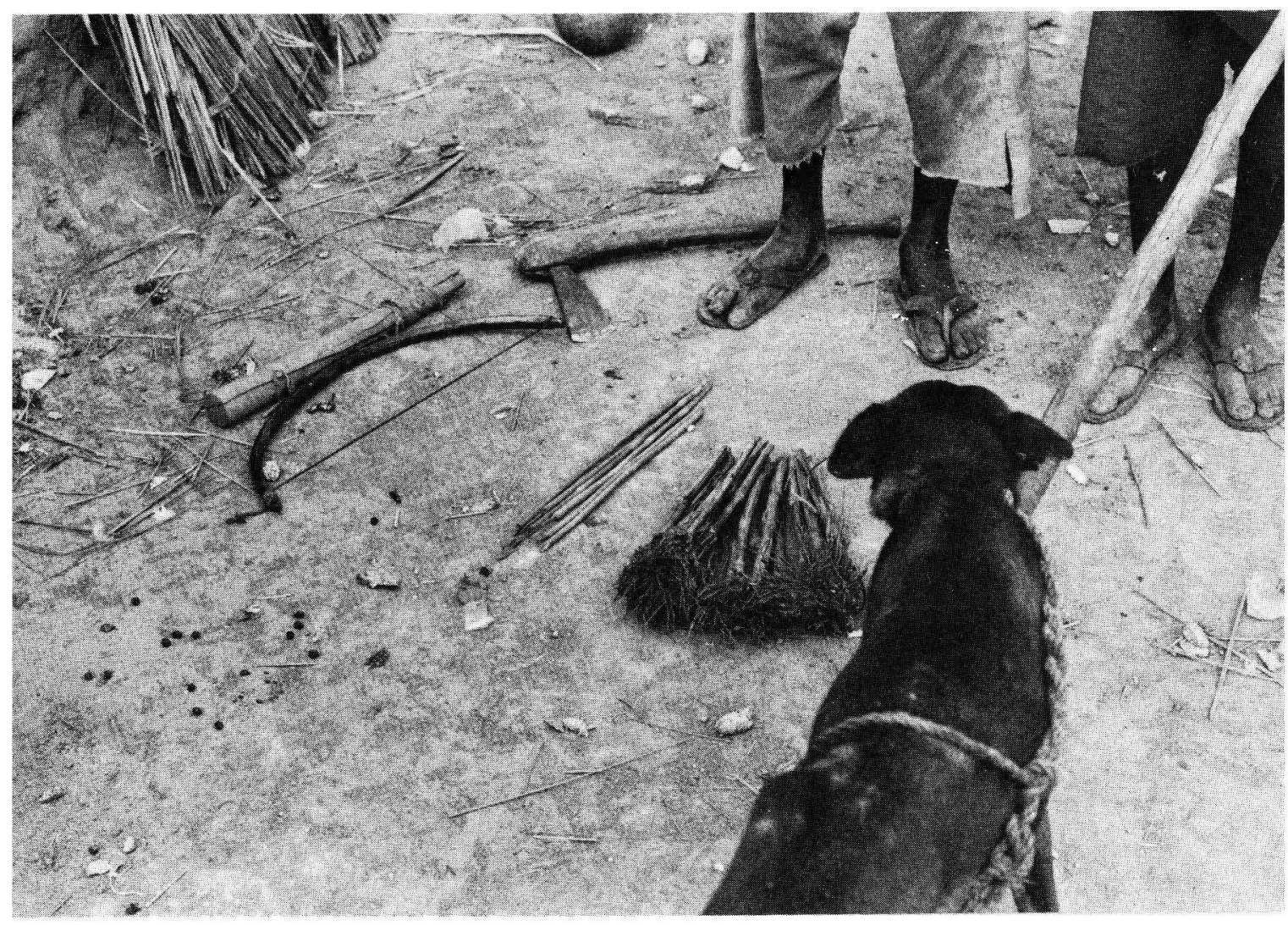

Photo 1. Le chien, avant le sacrifice, mis en présence des "affaires d'homme" du père défunt. 


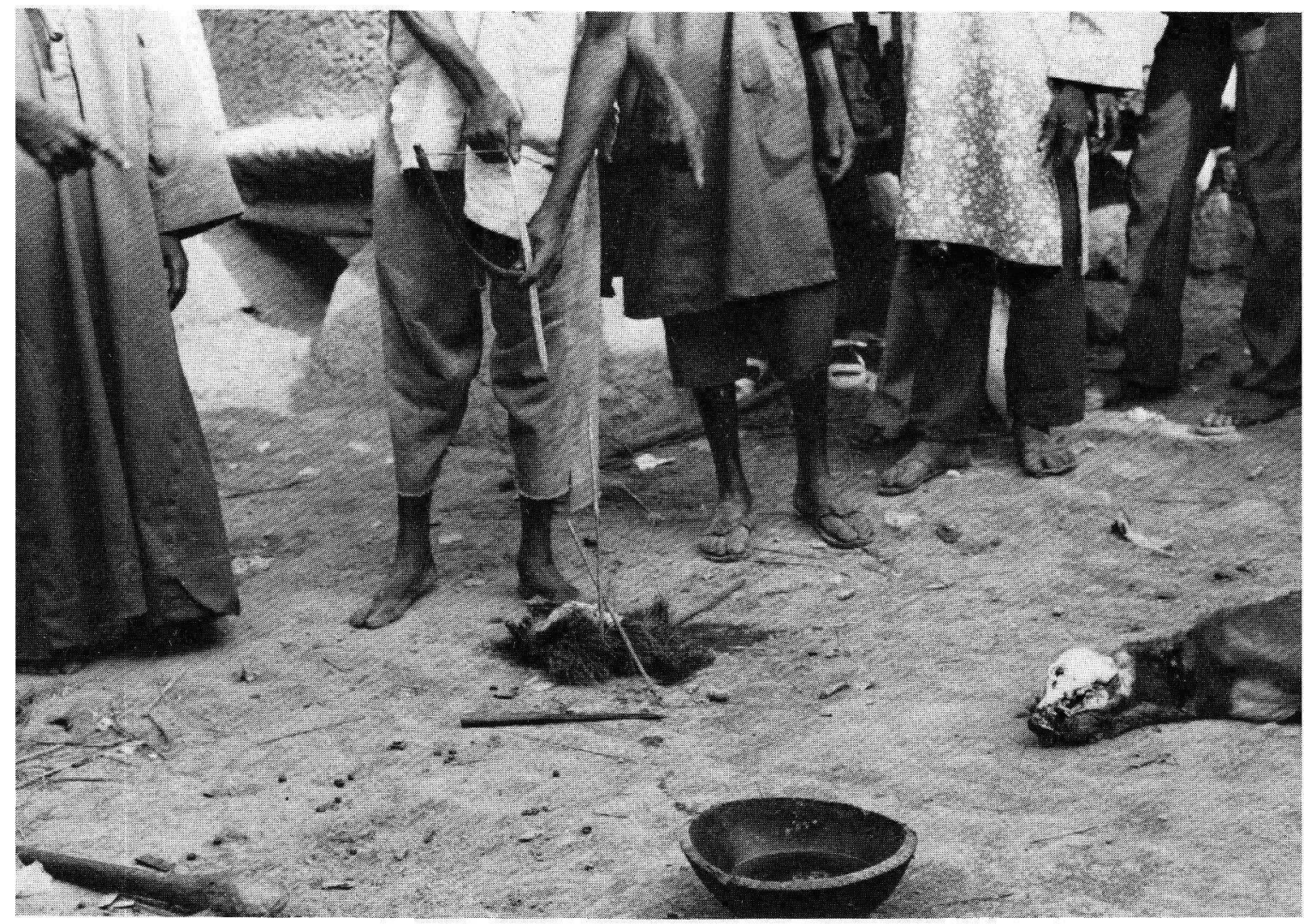

Photo 2. Le fils aîné sacrificateur décoche deux flèches sur des morceaux de corps du chien, posés sur des souches de mil. 


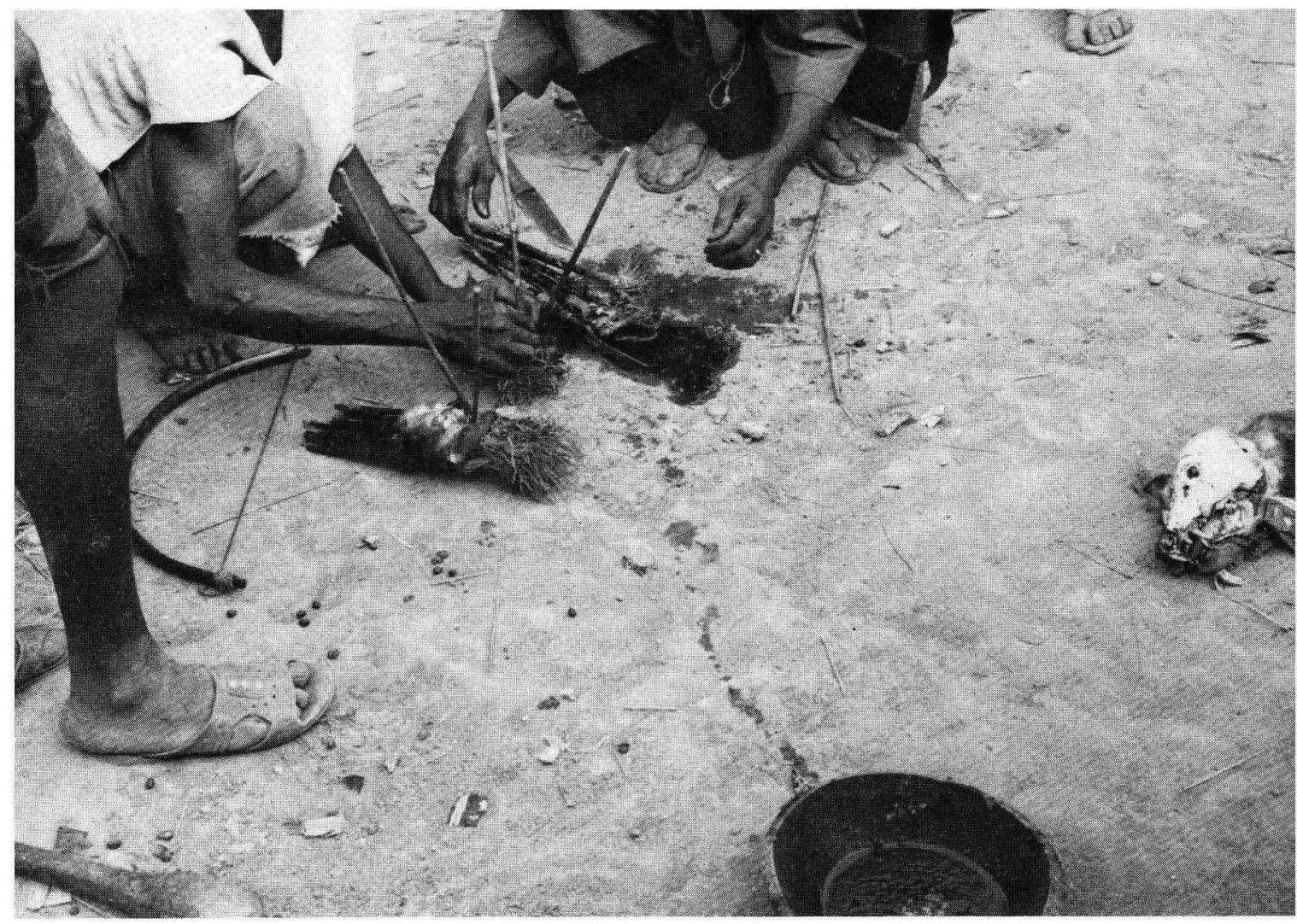

Photo 3. Le fils aîné reconstitue le "trophée de guerre" de son père défunt. 


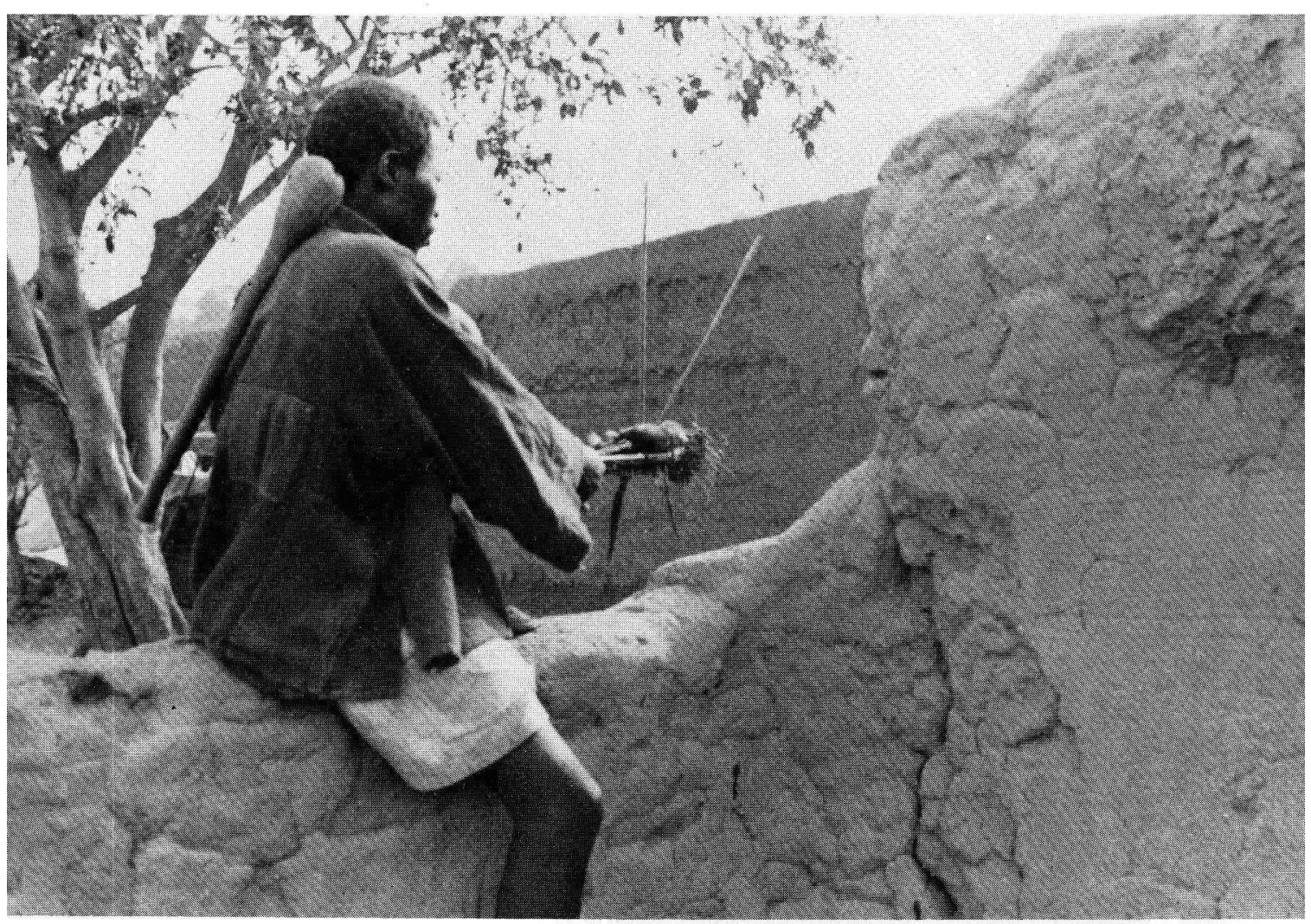

Photo 4. Un homme, étranger à $1 \mathrm{a}$ famille, sort 1e trophée de guerre de la maison paternelle par le mur de clôture. 


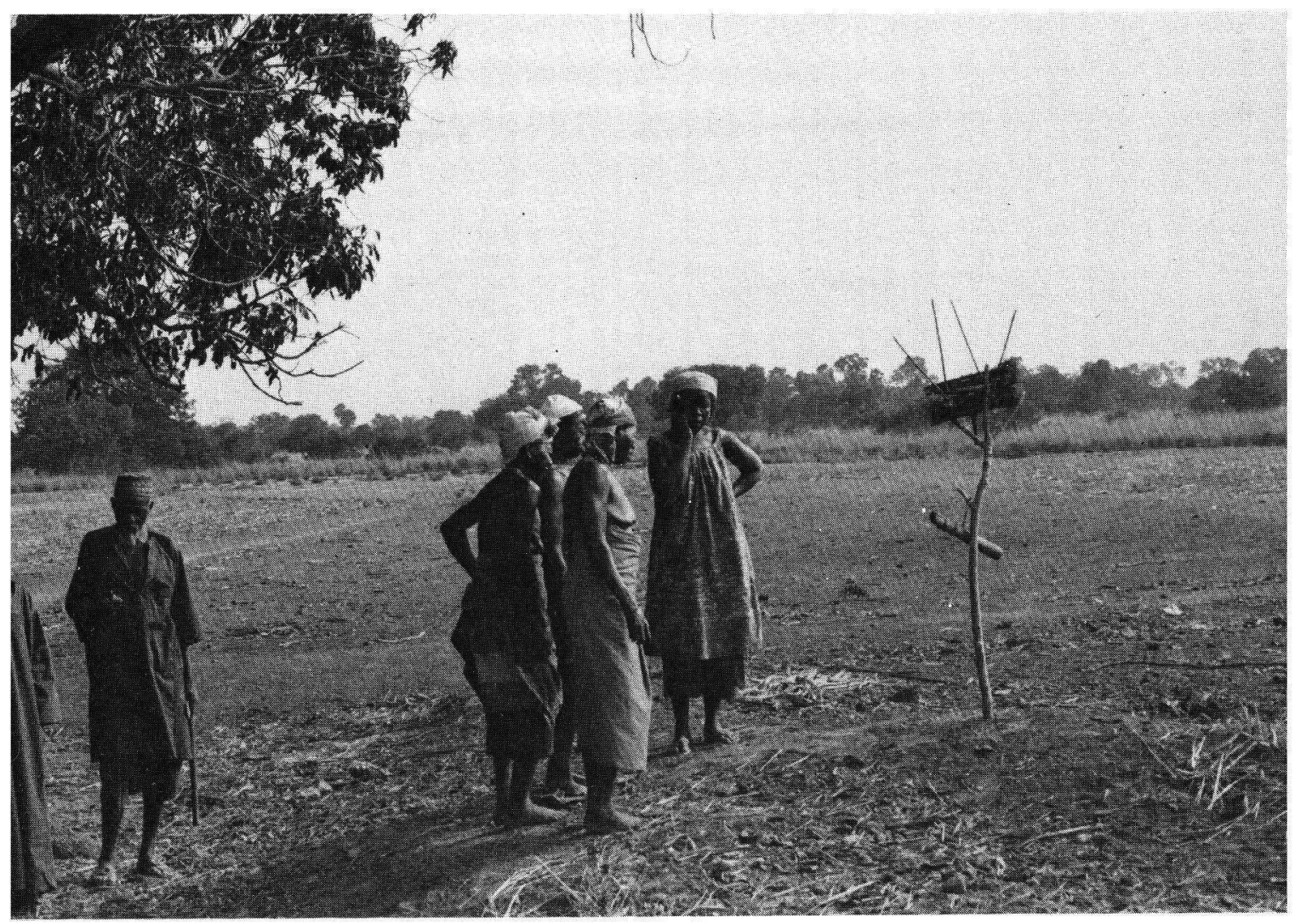

Photo 5. Les veuves face à "1'autel du meurtre" dressé sur le champ des ordures. 